1 The AAA+ chaperone VCP disaggregates Tau fibrils and generates

\title{
aggregate seeds
}

3
Itika Saha $^{1,2}$, Patricia Yuste-Checa ${ }^{1,2}$, Miguel Da Silva Padilha ${ }^{3,4}$, Qiang Guo ${ }^{5, \#}$, Roman Körner ${ }^{1}$, Hauke Holthusen ${ }^{1}$, Victoria A. Trinkaus ${ }^{1,5,6}$, Irina Dudanova ${ }^{3,4}$, Rubén FernándezBusnadiego $^{2,5,7,8}$, Wolfgang Baumeister ${ }^{5}$, David W. Sanders ${ }^{9,}$, Saurabh Gautam ${ }^{1, \S}$, Marc I. Diamond $^{9}$, F. Ulrich Hartl ${ }^{1,2,6,{ }^{*}}$ and Mark S. Hipp ${ }^{1,6,10,11^{*}}$

${ }^{1}$ Department of Cellular Biochemistry, Max Planck Institute of Biochemistry, Am Klopferspitz 18, 82152 Martinsried, Germany.

${ }^{2}$ Aligning Science Across Parkinson's (ASAP) Collaborative Research Network, Chevy Chase, MD, USA.

${ }^{3}$ Molecular Neurodegeneration Group, Max Planck Institute of Neurobiology, 82152 Martinsried, Germany.

${ }^{4}$ Department of Molecules - Signaling - Development, Max Planck Institute of Neurobiology, Am Klopferspitz 18, 82152 Martinsried, Germany.

${ }^{5}$ Department of Structural Molecular Biology, Max Planck Institute of Biochemistry, Am Klopferspitz 18, 82152 Martinsried, Germany.

${ }^{6}$ Munich Cluster for Systems Neurology (SyNergy), Munich, Germany.

${ }^{7}$ Institute of Neuropathology, University Medical Center Göttingen, 37099 Göttingen, Germany.

${ }^{8}$ Cluster of Excellence "Multiscale Bioimaging: from Molecular Machines to Networks of Excitable Cells" (MBExC), University of Göttingen, Germany.

${ }^{9}$ Center for Alzheimer's and Neurodegenerative Diseases, Peter O’Donnell Jr. Brain Institute, University of Texas Southwestern Medical Center, Dallas, 75390 Texas, USA. 
$24{ }^{10}$ School of Medicine and Health Sciences, Carl von Ossietzky University Oldenburg, 25 Oldenburg, Germany.

$26{ }^{11}$ Department of Biomedical Sciences of Cells and Systems, University Medical Center

27 Groningen, University of Groningen, Antonius Deusinglaan, 1, 9713 AV Groningen, The

28 Netherlands.

29 \# Present address: State Key Laboratory of Protein and Plant Gene Research, School of Life

30 Sciences and Peking-Tsinghua Center for Life Sciences, Peking University, Beijing 100871,

31 China.

$32 \ddagger$ Present address: Department of Chemical and Biological Engineering, Princeton University, 33 Princeton, NJ 08544, USA

$34 \S$ Present address: Boehringer Ingelheim International GmbH, 55216 Ingelheim, Germany and 35 ViraTherapeutics GmbH, 6063 Rum, Austria.

36

37 * To whom correspondence should be addressed:

38 uhartl@biochem.mpg.de (FUH); m.s.hipp@umcg.nl (MSH)

39

40 


\section{4}

\section{Abstract}

Amyloid-like aggregates of the microtubule-associated protein Tau are associated with several neurodegenerative disorders including Alzheimer's disease. The existence of cellular machinery for the removal of such aggregates has remained unclear, as specialized disaggregase chaperones are thought to be absent in mammalian cells. Here we show in cell culture and in neurons that the $\mathrm{AAA}+$ chaperone VCP is recruited to ubiquitylated Tau fibrils, resulting in their efficient disaggregation. Aggregate clearance depends on the functional cooperation of VCP with Hsp70 and the ubiquitin-proteasome machinery. Inhibition of VCP activity stabilizes large Tau aggregates, and is accompanied by a reduction in the amount of Tau species competent of prionlike aggregate seeding in recipient cells. Thus, disaggregation by VCP generates seeding-active Tau as byproduct. These findings identify VCP as a core component of the machinery for the removal of neurodegenerative disease aggregates and suggest that its activity can be associated with enhanced aggregate spreading in tauopathies.

\section{Introduction}

Deposition of amyloid-like Tau aggregates is a hallmark of devastating neurodegenerative disorders such as Alzheimer's disease and frontotemporal dementia ${ }^{1}$. In healthy neurons, Tau functions in microtubule (MT) assembly and stabilization by associating with MTs via its repeat domain (RD) consisting of three or four 31-32 residue imperfect repeats. Two hexapeptide motifs within the RD are critical for Tau aggregation, and the RD forms the structural core of disease-associated aggregates ${ }^{1}$, with several RD mutations underlying familial tauopathies ${ }^{2}$. Expression of human Tau mutants in mouse models recapitulates essential features of 
tauopathies including the formation of amyloid-like Tau deposits and neuronal loss ${ }^{3,4}$, indicating that Tau aggregation is central to neurodegeneration. Pathogenic Tau aggregates often exhibit the ability to induce aggregation in naïve cells through a mechanism of transcellular propagation that allows aggregate pathology to spread across brain regions ${ }^{5,6}$. Notably, pathological Tau aggregates and spreading are resolved upon lowering Tau levels, which is accompanied by improved neuronal health and extended lifespan ${ }^{7,8}$. However, the cellular mechanisms involved in the reversal, clearance and spread of Tau aggregates remain poorly understood.

While specialized chaperones of the AAA+ family in bacteria, yeast and plants have the ability to resolve amyloid-like aggregates ${ }^{9,10}$, direct homologues of these hexameric disaggregases have not been identified in mammalian cells. Instead, disaggregation in higher eukaryotes is mainly attributed to the Hsp70 chaperone machinery ${ }^{11-14}$. The human Hsp70Hsp40-Hsp110 chaperone system efficiently dissociates Tau and $\alpha$-synuclein fibrils in vitro ${ }^{15-17}$ independent of AAA+ disaggregases that cooperate with the Hsp70 system in yeast and bacteria to achieve disaggregation ${ }^{9}$. The eukaryotic AAA + ATPase valosin-containing protein (VCP) exerts ATP-dependent protein unfolding activity ${ }^{18,19}$ and has been proposed to resolve protein aggregates $^{20,21}$ and certain condensates such as stress granules ${ }^{22,23}$. VCP facilitates protein turnover via the ubiquitin-proteasome system ${ }^{24,25}$, in addition to sustaining functional autophagy $^{26}$. Indeed, VCP mutations have been associated with aggregate deposition disorders such as vacuolar tauopathy and inclusion body myopathy associated with Paget disease of bone and frontotemporal dementia (IBMPFD) $)^{20,27-29}$. Accumulation of Tau aggregates in vacuolar tauopathy was proposed to be a consequence of diminished ATPase activity of mutant VCP $(\mathrm{D} 395 \mathrm{G})^{20}$. IBMPFD-associated VCP mutants exhibit increased basal ATP hydrolysis and unfolding activity ${ }^{30,31}$, altered interactions with cofactors ${ }^{32,33}$ and perturbed autophagic 
86 function ${ }^{34}$. Whether any of these mutations influence the clearance of pre-formed fibrillar Tau 87 aggregates in cells is not known.

Here we provide direct evidence in a cell culture model and in primary murine neurons that VCP disaggregates amyloid-like Tau fibrils in a ubiquitin and proteasome-dependent manner, with the Hsp70 chaperone system contributing to aggregate clearance. This function of VCP is not detectably perturbed by pathogenic VCP mutations. Although disaggregation by VCP is coupled to proteasomal degradation, intermediates of the disaggregation process escape proteolysis and are a source of seeding-competent Tau species.

\section{Results}

To investigate the ability of cells to clear Tau aggregates, we used HEK293 cells stably expressing TauRD-Y (P301L/V337M), a mutant of the amyloid-forming repeat domain of $\mathrm{Tau}^{35,36}$ fused to YFP via a flexible linker ${ }^{37}$ (Fig. 1a). TauRD-Y is soluble and diffusely distributed in TauRD-Y cells, but the extracellular addition of Tau aggregates isolated from tauopathy brain tissue or generated in vitro induces its aggregation via template-based seeding, leading to formation of aggregates that are stably propagated for weeks ${ }^{37,38}$ (Fig. 1b). Using TauRD-Y aggregate seeds ${ }^{37}$, we generated a cell line (TauRD-Y*) in which phosphorylated TauRD-Y accumulated in cytosolic inclusions $0.5-5 \mu \mathrm{m}^{2}$ in size that stained with the amyloidspecific dye Amylo-Glo ${ }^{39}$ (Fig. 1c, Supplementary Fig. 1a-c). Analysis of the inclusions in intact TauRD-Y* cells by cryo-electron tomography revealed TauRD-Y fibrils of $\sim 18 \mathrm{~nm}$ diameter, which were distinguishable from cytoskeletal structures (Fig. 1d) and consistent with the structures of fibrillar Tau in tauopathy patient brain ${ }^{40-44}$. Thus, TauRD forms amyloid-like 
108 fibrillar aggregates in TauRD-Y* cells. TauRD-Y aggregates were also able to induce aggregates

109 of full-length Tau fused to YFP (FLTau-Y). These aggregates reacted with the AT-8 antibody

110 specific for phosphorylation at serine 202 and threonine 205 (epitopes not present in TauRD)

111 (Fig. 1a, Supplementary Fig. 1d,e), which has been used previously to detect paired helical

112 filaments ${ }^{45,46}$.

\section{Proteasomal clearance of Tau aggregates}

115 Soluble TauRD-Y was efficiently degraded in TauRD-Y cells upon inhibition of protein

116 synthesis with cycloheximide (CHX) (Supplementary Fig. 1f). CHX treatment also led to partial

117 clearance of TauRD inclusions in TauRD-Y* cells (Fig. 1e, Supplementary Fig. 1f,g). To avoid

118 global inhibition of protein synthesis, we employed cells in which the expression of TauRD-Y is

119 controlled with a Tet-regulated promoter (Tet-TauRD-Y and Tet-TauRD-Y* cells) ${ }^{37}$

120 (Supplementary Fig. 2a). Addition of doxycycline resulted in clearance of TauRD-Y inclusions

121 and insoluble TauRD-Y protein ( $\mathrm{t}_{1 / 2} \sim 12 \mathrm{~h}$ ) (Supplementary Fig. 2a-d). The amount of insoluble

122 TauRD-Y decreased faster than the level of soluble TauRD-Y (Supplementary Fig. 2d),

123 consistent with aggregate material being solubilized prior to degradation. Moreover, inhibition of

124 TauRD-Y synthesis resulted in a time dependent reduction of inclusion size and number per cell

125 (Fig. 1f). Thus, the cells are able to efficiently dissociate and degrade amyloid-like TauRD-Y

126 aggregates.

Addition of the selective proteasome inhibitor Epoxomicin or siRNA-mediated

128 downregulation of the proteasome component PSMD11 stabilized aggregated TauRD-Y upon

129 doxycycline shut-off and prevented aggregate clearance (Supplementary Fig. 3a-e). Proteasome

130 inhibition also stabilized soluble TauRD-Y in Tet-TauRD-Y cells ${ }^{47}$ (Supplementary Fig. 3a), but 
131 did not lead to de novo Tau aggregation ${ }^{48}$ (Supplementary Fig. 4h). Hence, the persistence of

132 TauRD-Y aggregates upon proteasome inhibition is due to stabilization of pre-existing

133 aggregates. In contrast, inhibition of lysosomal degradation with Bafilomycin A1 (confirmed by

134 increased levels of LC3-II) or of autophagy with 3-methyladenine was without effect on the

135 levels of total or aggregated TauRD-Y protein in the cellular model used (Supplementary Fig.

136 3a-c). Downregulation of autophagy components ATG5/7 supported this conclusion

137 (Supplementary Fig. 3d,e). Thus, TauRD-Y aggregates are degraded in a proteasome-dependent, 138 autophagy-independent manner.

\section{Tau disaggregation requires VCP}

141 Proteins must generally be unfolded to access the catalytic center of the $20 \mathrm{~S}$ proteasome. Thus, 142 prior to degradation, aggregated proteins need to undergo disaggregation ${ }^{49}$. To identify the 143 cellular machinery involved in TauRD-Y disaggregation, we performed an interactome analysis 144 of aggregated TauRD-Y by quantitative mass spectrometry. We identified the AAA+ ATPase

$145 \mathrm{VCP}$ as one of the most highly enriched interactors of aggregated TauRD-Y, along with the 146 ubiquitin-binding VCP cofactors UFD1L, NPLOC4 and NSFL1C, and multiple subunits of the

147 26S proteasome (Fig. 2a, Supplementary Table 1). Hsp70 was detected in the proteomic analysis

148 but was not enriched on aggregated TauRD-Y. Co-localization of VCP and its cofactors with

149 TauRD-Y aggregates was confirmed by fluorescence microscopy (Fig. 2b, Supplementary Fig. $1504 a-c)$.

152 in different cellular contexts ${ }^{18,50}$. To assess whether VCP is involved in TauRD-Y

153 disaggregation, we inhibited VCP in cells using NMS-873, a small molecule allosteric inhibitor 
154 of the VCP ATPase ${ }^{51}$. Similar to proteasome inhibition, NMS-873 blocked the clearance of

155 TauRD-Y aggregates when TauRD-Y synthesis was stopped with doxycycline (Fig. 2c,d).

156 Likewise, the aggregates were stabilized when VCP was inhibited using CB-5083

157 (Supplementary Fig. 4d), a competitive inhibitor of ATP binding in the D2 ATPase domain of

$158 \mathrm{VCP}^{52}$, or down-regulated with siRNA (Supplementary Fig. 4e,f). VCP inhibition during

159 ongoing TauRD-Y synthesis resulted in a significant increase in inclusion size (Supplementary

160 Fig. 4g), suggesting that the inclusions exist at a dynamic equilibrium between formation and

161 disaggregation. No aggregation of soluble TauRD-Y was detected after treating cells with NMS-

162873 or VCP siRNA (Supplementary Fig. 4 h,i). VCP down-regulation caused a marginal increase

163 in the level of soluble TauRD-Y in Tet-TauRD-Y cells, but did not result in a significant

164 stabilization after doxycycline addition (Supplementary Fig. 4j). In contrast, aggregate-

165 containing Tet-TauRD-Y* cells treated with VCP siRNA accumulated significantly higher

166 amounts of TauRD-Y both in the absence or presence of doxycycline, indicating an aggregate-

167 specific role of VCP (Supplementary Fig. 4j).

168 Importantly, VCP also co-localized with aggregates of full-length Tau (FLTau-Y) in

169 FLTau-Y* cells (Supplementary Fig. 4k), and VCP or proteasome inhibition prevented the

170 clearance of FLTau-Y aggregates (Supplementary Fig. 41,m), recapitulating the behavior of

171 TauRD. To exclude a possible role of the YFP tag on Tau in VCP-mediated disaggregation, we

172 generated HEK293T cells stably expressing non-tagged full-length Tau (FLTau) and myc-tagged

173 Tau repeat domain (TauRD) under a Tet-regulated promoter (Tet-FLTau, Tet-FLTau* and Tet-

174 TauRD, Tet-TauRD* cells). Similar to FLTau-Y aggregates in FLTau-Y* cells, FLTau

175 aggregates in Tet-FLTau* cells were phosphorylated at serine 202 and threonine 205 and

176 colocalized with VCP (Fig. 2e, Supplementary Fig. 4n). FLTau and TauRD aggregates were 
resolved in a VCP and proteasome-dependent manner when Tau synthesis was halted by adding doxycycline (Fig. 2f, Supplementary Fig. 4o).

We next tested whether VCP also modulates Tau aggregation in neurons. Mouse primary neurons were transduced to express soluble TauRD-Y (Fig. 3a,b). Upon seeding with TauRD $\operatorname{aggregates}^{37}$, we observed the formation of multiple inclusions of intracellular TauRD-Y (Fig. 3b). Cryo-electron tomography of TauRD-Y inclusions in aggregate-containing neurons revealed fibrillar aggregates similar to the aggregates in TauRD-Y* cells (Fig. 3c). The lower cytosolic density of neurons allowed the observation that the TauRD-Y fibrils were coated with globular domains consistent with the presence of YFP on TauRD (Supplementary Fig. 4p), as previously observed for other amyloidogenic proteins fused to fluorescent protein $^{53,54}$. Aggregate seeding in neurons was accompanied by a $\sim 40 \%$ decrease in cell viability (Fig. $3 \mathrm{~d}$ ). Most of the neuronal TauRD-Y inclusions stained positive for VCP (Fig. 3b). Treatment with the VCP inhibitor NMS-873 for $4 \mathrm{~h}$ caused a massive accumulation of TauRD aggregates in seeded neurons, in some cases occupying most of the cell body area (Fig. 3e). No inclusions were observed in unseeded cells upon VCP inhibition (Fig. 3e). These results demonstrate that VCP functions in TauRD-Y disaggregation in neurons.

\section{Disaggregation depends on substrate ubiquitylation}

Ubiquitylation of VCP substrates, particularly the formation of lysine 48 (K48) linked polyubiquitin chains, is required for VCP recruitment ${ }^{18,19,30,50}$. We therefore analyzed immunoprecipitates of TauRD-Y for the presence of ubiquitin. Only in TauRD-Y* cells containing aggregated TauRD-Y was the protein detectably modified by the addition of 1 to 4 ubiquitin molecules (Fig. 4a). Analysis with a K48-specific antibody verified the presence of 
K48-linked ubiquitin (Fig. 4a). Immunofluorescence imaging also showed that the TauRD-Y

201 aggregates stained positive for K48-linked ubiquitin chains (Fig. 4b, Supplementary Fig. 5a),

202 while K63-linked ubiquitin was not detectable (Supplementary Fig. 5a). Likewise, the TauRD-Y

203 inclusions in primary neurons colocalized with poly-ubiquitin chains (Supplementary Fig. 5b).

204 K48-linked ubiquitin signal was also observed on the aggregates of untagged FLTau and TauRD

205 (Supplementary Fig. 5c).

Inhibition of the ubiquitin-activating enzyme E1 with the specific inhibitor MLN7243 ${ }^{55}$

207 efficiently blocked ubiquitin conjugation (Supplementary Fig. 6a). TauRD-Y inclusions were

208 still present but were no longer ubiquitin K48-reactive (Fig. 4b, Supplementary Fig. 6b). VCP

209 was not recruited to these aggregates (Fig. 4c, Supplementary Fig. 6c), and both disaggregation

210 and degradation of TauRD-Y in Tet-TauRD-Y* cells were blocked (Fig. 4d, Supplementary Fig.

211 6d). MLN7243 treatment also prevented the degradation of soluble TauRD-Y to a degree similar

212 to proteasome inhibition (Supplementary Fig. 6e). Together these data show that VCP

213 recruitment requires ubiquitylation of aggregated Tau, followed by disaggregation and

214 remodeling to species that are accessible for proteasomal degradation.

\section{Functions of VCP and Hsp70 in disaggregation}

217 Disaggregation of both heat stress-induced and amyloid-like aggregates in mammalian cells has

218 been assigned to the Hsp70 chaperone system ${ }^{11-13,16}$. Our findings raised the possibility of a

219 functional cooperation between VCP and Hsp70 in these processes. To determine whether VCP

220 participates in dissolving heat-induced aggregates, we expressed the metastable protein firefly

221 luciferase (Fluc) fused to GFP in HEK293 cells. Heat stress at $43{ }^{\circ} \mathrm{C}$ for $2 \mathrm{~h}$ combined with

222 proteasome inhibition resulted in the formation of large $(\sim 2-3 \mu \mathrm{m})$ Fluc-GFP inclusions ${ }^{56}$ 
223 (Supplementary Fig. 7a). Unlike the TauRD-Y inclusions, the Fluc-GFP aggregates did not stain 224 with an amyloid-specific dye (Supplementary Fig. 7a), suggesting that they were amorphous in 225 structure. The Fluc-GFP aggregates were ubiquitin-negative and did not co-localize with VCP 226 (Supplementary Fig. 7b-c). Accordingly, VCP inhibition with NMS-873 did not interfere with 227 disaggregation (Supplementary Fig. 7d), arguing against a role of VCP in this process. However, 228 inhibition of the ATPase activity of Hsp70 with the inhibitor VER-155008 ${ }^{57}$ prevented Fluc-GFP 229 disaggregation (Supplementary Fig. 7d), confirming the role of the Hsp70 system in 230 disaggregation.

To investigate whether Hsp70 participates in TauRD-Y disaggregation, we treated Tet232 TauRD-Y* cells with VER-155008 or with NMS-873 and stopped TauRD-Y synthesis with 233 doxycycline. VCP inhibition stabilized both large $\left(>1.5 \mu \mathrm{m}^{2}\right)$ and small $\left(<1.5 \mu \mathrm{m}^{2}\right)$ TauRD-Y 234 inclusions (Supplementary Fig. 8a-b). In contrast, Hsp70 inhibition stabilized large aggregates 235 only partially and resulted in a marked accumulation of small inclusions, consistent with VCP 236 acting before Hsp70 in the disaggregation process (Supplementary Fig. 8a-b). These findings 237 suggested that Hsp70 cooperates with VCP in disaggregation, either by dissociating fragments 238 generated by VCP and/or by preventing re-aggregation of TauRD liberated from inclusions by 239 VCP. Since Hsp70 was not enriched on TauRD-Y aggregates in the proteomic analysis 240 (Supplementary Table 1), its interaction with TauRD may be transient.

\section{Effects of VCP mutants on Tau disaggregation}

243 Point mutations in VCP are associated with dominantly inherited disorders such as Inclusion

244 body myopathy with Paget disease of bone and frontotemporal dementia (IBMPFD) ${ }^{26}$ and 245 vacuolar tauopathy ${ }^{20}$. These mutations lead to a dominant negative loss or alteration of VCP 
246 function, presumably due to the oligomeric nature of $\mathrm{VCP}^{58,59}$. The mutation $\mathrm{D} 395 \mathrm{G}$ (DG),

247 associated with vacuolar tauopathy is located in the D1 ATPase domain of VCP (Fig. 5a). It has

248 been reported to have a mildly reduced capacity to disaggregate Tau fibrils in an in vitro system, 249 due to a $\sim 30 \%$ reduced ATPase activity ${ }^{20}$. The IBMPFD mutations, A232E (AE) and R155H

$250(\mathrm{RH})$, are located in the D1 ATPase domain and in the N-domain, respectively, and are

251 associated with enhanced ATPase activity compared to wild type (WT) VCP ${ }^{31}$ (Fig. 5a). We

252 tested whether these mutations impair Tau disaggregation in our cellular model using the

253 ATPase defective VCP double-mutant E305Q/E578Q (EQ/EQ) ${ }^{60}$ (Fig. 5a) as a control. The

254 mutant proteins, carrying a C-terminal myc-tag, were transiently overexpressed in Tet-TauRD-

$255 \mathrm{Y}^{*}$ cells for $24 \mathrm{~h}$ and then TauRD-Y synthesis was stopped with doxycycline to observe

256 disaggregation. Note that mutant VCP was expressed in cells containing pre-formed aggregates

257 to exclude a potential role of VCP in aggregate seeding ${ }^{61}$. The myc-tagged mutant proteins were

258 present in hexamers that migrated on native PAGE like WT VCP (Supplementary Fig. 9a) and

259 colocalized with TauRD-Y aggregates (Fig. 5b).

As expected, expression of the ATPase defective VCP (EQ/EQ) effectively prevented

261 TauRD-Y aggregate clearance, even though the expression levels of this mutant were relatively

262 low when compared with the other constructs (Fig. 5c,d). Moreover, the TauRD-Y aggregates

263 increased in size upon VCP (EQ/EQ) expression as observed previously for inhibition of VCP

264 activity by NMS-873 (Supplementary Fig. 4g), presumably reflecting a shift of soluble TauRD to

265 the aggregates (Supplementary Fig. 2a). However, none of the disease-related VCP mutants,

266 including the vacuolar tauopathy mutant DG, when expressed at the indicated levels, detectably

267 stabilized TauRD-Y aggregates (Fig. 5b-d). Similar results were obtained when the presence of

268 aggregates was specifically analyzed in cells expressing the mutant VCP proteins by 
immunofluorescence (Supplementary Fig. 9b). In conclusion, the effect of the VCP disease mutations on disaggregation, if any, is only mild, suggesting that inhibition of aggregate clearance may not be the primary mechanism by which these mutations cause disease.

\section{VCP generates Tau species capable of seeding aggregation}

Progression of tauopathies and other neurodegenerative diseases is thought to be mediated by aggregate spreading across brain regions through a prion-like seeding mechanism ${ }^{5,6}$. We speculated that the disaggregation activity of VCP could modulate the levels of aggregate species that are able to induce the aggregation of soluble Tau in recipient cells. To address this possibility, we measured the presence of seeding-competent TauRD species by FRET in cells expressing TauRD-mTurquoise2 and TauRD-Y (TauRD-TY cells) ${ }^{62}$ (Fig. 6a). Addition of aggregate-containing total lysates from control TauRD-Y* cells induced TauRD aggregation in reporter cells (Supplementary Fig. 10a-b). Strikingly, treatment of TauRD-Y* cells with the VCP inhibitor NMS-873 reduced the seeding capacity of lysates by more than $50 \%$, when equivalent amounts of TauRD-Y were compared (Fig. 6b). In contrast, such a reduction in seeding was not observed when cells were treated with proteasome inhibitor (Epoxomicin) or Hsp70 inhibitor (VER-155008) (Fig. 6b). However, treatment with the E1 enzyme inhibitor MLN7243, which prevented VCP recruitment to the aggregates (Fig. 4c), also caused a $\sim 50 \%$ reduction of FRET positive TauRD-TY reporter cells. Similar effects were observed when lysates from TauRD-Y* cells transiently expressing VCP EQ/EQ were used. In contrast, expression of VCP DG did not reduce seeding (Fig. 6c, Supplementary Fig. 10c). Thus, VCP-mediated disaggregation generates seeding-active TauRD-Y species. 
To characterize the seeding competent material in the presence and absence of VCP

292 function, we fractionated lysates from TauRD-Y* cells by size-exclusion chromatography.

293 Inclusions $>0.2 \mu \mathrm{m}$ were removed by filtration. The majority of the remaining TauRD-Y $(\sim 70 \%)$

294 fractionated at a high molecular weight (HMW) of $\geq 40 \mathrm{MDa}$ in the void volume of the column.

295 The remainder fractionated at a low molecular weight (LMW), equivalent to the position of

296 soluble TauRD-Y from TauRD-Y cells (Fig. 6d). Both fractions isolated from TauRD-Y* cells

297 were seeding competent, but the specific seeding activity of HMW TauRD-Y (\% FRET positive

298 cells per ng TauRD-Y) was $\sim 10$-fold higher than that of the LMW fraction (Supplementary Fig.

299 10d). Treatment with VCP inhibitor NMS-873 strongly reduced the total amount of TauRD-Y

300 species $<0.2 \mu \mathrm{m}$, consistent with the reduced seeding activity after VCP inhibition. Moreover,

301 the ratio between HMW and LMW peaks was reversed as the former was decreased by $\sim 80 \%$

302 and the latter by only $25 \%$ (Fig. 6d). However, the specific seeding activity of TauRD in both

303 fractions remained unchanged (Supplementary Fig. 10d), suggesting that VCP inhibition reduces

304 the amount of seeds but not their intrinsic seeding potency. Together these results demonstrate

305 that the disaggregation activity of VCP increases the available pool of seeding competent TauRD

306 species.

\section{Discussion}

309 Metazoa do not possess a homologue of the AAA+ ATPase Hsp104 responsible for protein

310 disaggregation in bacteria, fungi and plants ${ }^{10,63}$. Instead, dissociation of large protein aggregates,

311 including amyloid fibrils, in animal cells is generally ascribed to the Hsp70/Hsp110/Hsp40

312 chaperone system ${ }^{10,12-14,16}$. Here we provide evidence that the AAA+ ATPase VCP functions in

313 disaggregating amyloid fibrils of Tau in human cells and primary mouse neurons (Fig. 7). VCP 
314 is distinct from Hsp104 in that it requires the target aggregate to be ubiquitylated, a critical

315 element of control to ensure specificity and avoid dissolution of functional protein assemblies ${ }^{64}$.

316 Consistent with such a control function, ubiquitylation likely occurs after aggregate formation as

317 an essential prerequisite for disaggregation (Fig. 7). The E3 ubiquitin ligases involved in this

318 process remain to be identified. Moreover, aggregate ubiquitylation ensures that disaggregation

319 by VCP is coupled to degradation by the $26 \mathrm{~S}$ proteasome. Additionally, the proteasomal $19 \mathrm{~S}$

320 ATPase may contribute to disaggregation, consistent with its ability to fragment fibrils in vitro ${ }^{65}$.

321 The Hsp70 chaperone system is required for the overall efficiency of the reaction, either by

322 further dissociating aggregate fragments produced by VCP action or by preventing re-

323 aggregation of Tau molecules that have been liberated from the fibrils (Fig. 7). As the smaller

324 TauRD aggregates that accumulated upon Hsp70 inhibition were no longer VCP positive,

325 disaggregation by VCP may allow Hsp70 to access aggregates of non-ubiquitylated Tau. This

326 possibility is consistent with the reported ability of the Hsp70 system to disaggregate Tau

327 aggregates in vitro ${ }^{15}$.

Support for the physiological relevance of VCP in antagonizing amyloid aggregation is

329 provided by mutations in VCP that are associated with the deposition of ubiquitylated aggregates

330 in neurodegenerative diseases such as vacuolar tauopathy and IBMPFD ${ }^{20,28,58}$. However, the

331 vacuolar tauopathy-associated VCP mutation $\mathrm{D} 395 \mathrm{G}^{20}$ and the IBMPFD mutations A232E and

$332 \mathrm{R} 155 \mathrm{H}^{26}$ did not detectably impair VCP-mediated Tau disaggregation in our cellular model.

333 Although even a small inhibitory effect on disaggregation may contribute to aggregate pathology

334 in neurons over decades, the disease mutations may alternatively affect other steps during

335 aggregate formation, including aggregate seeding and Tau degradation in cooperation with the

336 proteasome. Indeed, an increase in intracellular aggregation is observed when VCP is inhibited 
in recipient cells at the time of seeding ${ }^{61}$ or when VCP D395G is expressed in the recipient cells $^{20}$. Note that we introduced the mutant VCP proteins in cells containing preexistent Tau aggregates to exclude a potential role of VCP in the process of aggregate seeding.

Our finding that clearance of Tau aggregates by VCP generates smaller seeding competent species as a byproduct (Fig. 7) provides a plausible explanation for how VCP can exert both neuroprotective and neurotoxic effects. Indeed, overexpression of a VCP homologue in a Drosophila model of polyglutamine protein aggregation hastened the degenerative phenotype ${ }^{66}$. Transcellular aggregate spreading has been recognized as a major driver of neurodegenerative disease progression ${ }^{5,6,67}$, and generation of seeding competent species may be an inevitable consequence of amyloid clearance mechanisms via disaggregation, not only by VCP but also by the Hsp70 system ${ }^{15}$. We note, however, that in contrast to inhibition of VCP, neither Hsp70 nor proteasome inhibition had a significant effect on the generation of seeding competent Tau species in our model, suggesting that their function is not directly coupled to seed production.

VCP-mediated aggregate disassembly followed by proteasomal degradation provides an important alternative to autophagy as a mechanism for the elimination of terminally aggregated proteins. Based on our results, both activation and inhibition of this pathway may have beneficial effects dependent on the specific disease context. Non-human AAA + ATPases with augmented disaggregase activity are currently being developed with the aim to reverse pathogenic protein aggregation ${ }^{68,69}$. Boosting cellular aggregate clearance, perhaps in combination with proteasome activation $^{70}$, may offer a potential therapeutic strategy as long as the production of seeding competent species can be controlled. 
a

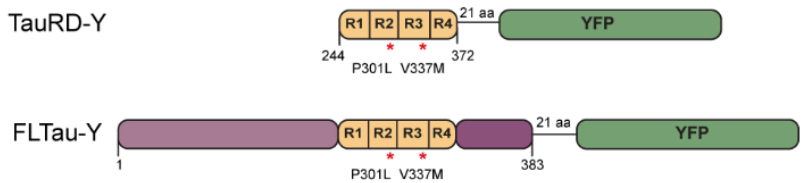

b

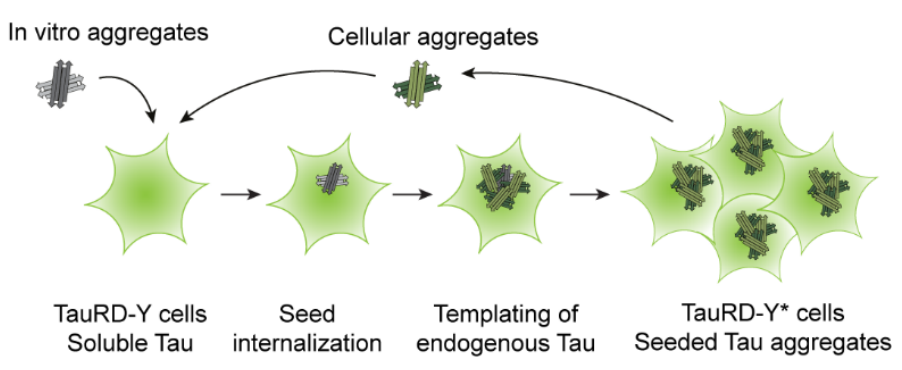

d

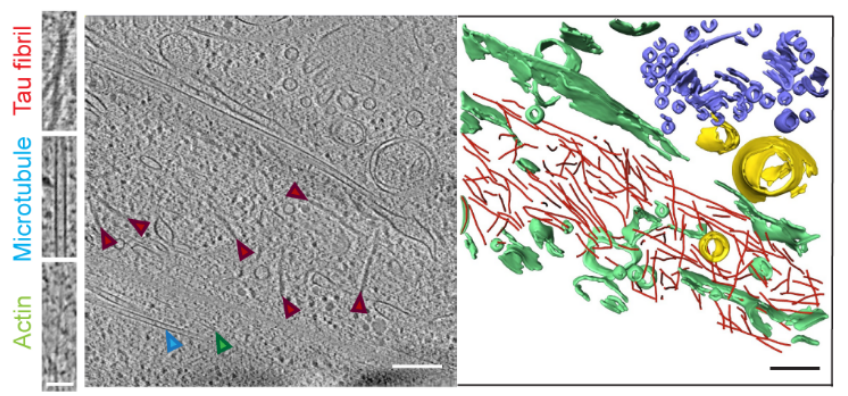

$\mathbf{f}$

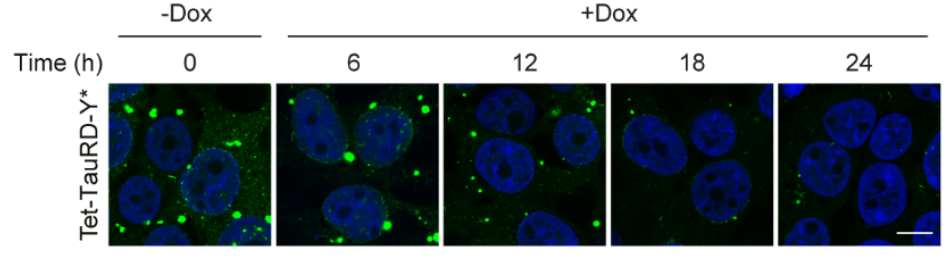

C

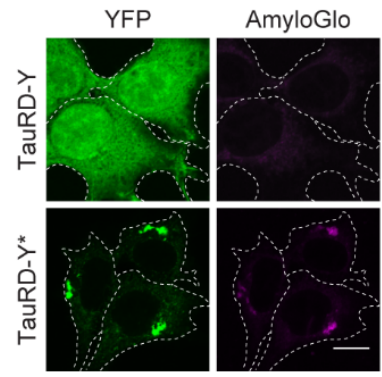

e
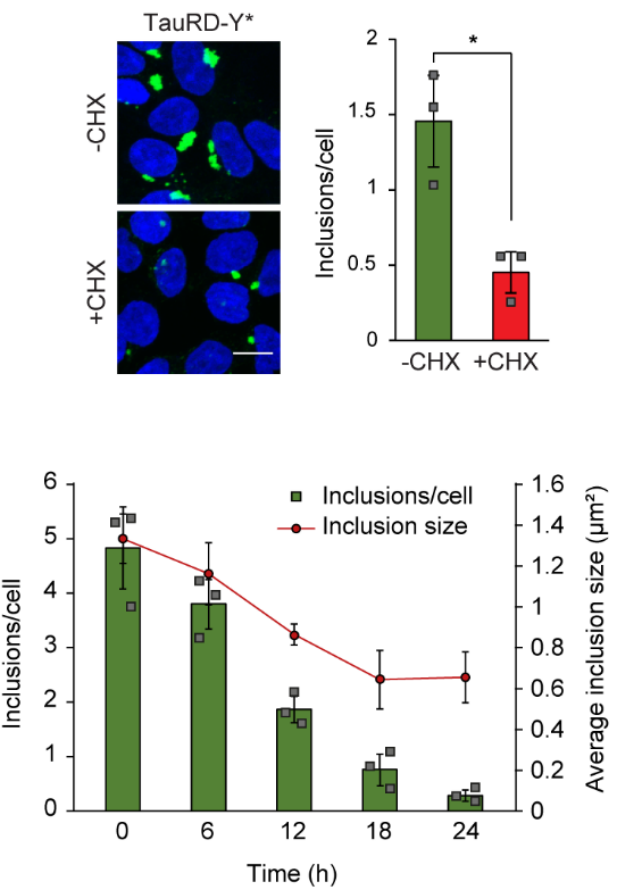

Fig. 1 TauRD-Y forms amyloid-like aggregates that are cleared from cells. a Schematic

364 representation of Tau constructs used in this study. TauRD-Y, the repeat domain, and FLTau-Y,

365 the 0N4R Tau isoform of full-length (FL) Tau with two frontotemporal dementia-associated 
mutations, P301L and V337M, fused to YFP via 21 amino acid (aa) linkers. b Schematic representation of aggregate seeding. Extracellular addition of preformed Tau aggregates induces

368 templating of intracellular Tau into aggregates that propagate with cell division. Aggregate seeds 369 may be generated in vitro or be contained in cell lysate. TauRD-Y, naïve cells containing soluble 370 TauRD-Y; TauRD-Y*, cells containing TauRD-Y aggregates. c Staining of TauRD-Y and 371 TauRD-Y* cells with the amyloid-specific dye Amylo-Glo (magenta). White dashed lines 372 indicate cell boundaries. Scale bar, $10 \mu \mathrm{m}$. d TauRD-Y aggregates are fibrillar in structure. Left, 373 a $1.7 \mathrm{~nm}$ thick tomographic slice of a TauRD inclusion from TauRD-Y* cells is shown. Red, 374 blue and green arrowheads indicate representative TauRD-Y fibril, microtubule and actin, 375 respectively. Right, 3D rendering of corresponding tomogram showing TauRD-Y fibrils (red), 376 Golgi (purple), mitochondria (yellow) and ER (green). Scale bars, $200 \mathrm{~nm}$, inset 40nm.

377 e Aggregate clearance. Left, TauRD-Y* cells treated for $24 \mathrm{~h}$ with cycloheximide (CHX; 50

$378 \mu \mathrm{g} / \mathrm{mL}$ ) where indicated. Nuclei were counterstained with DAPI (blue). Scale bar, $10 \mu \mathrm{m}$. Right, 379 quantification of TauRD-Y foci. Mean \pm s.d.; $n=3 ; 500-600$ cells analyzed per experiment; $380 * p<0.05(p=0.0151)$ from two-tailed Student's paired t-test. $\mathbf{f}$ Left, representative images of Tet381 TauRD-Y* cells treated with Dox for the indicated times. Right, quantification of inclusions per 382 cell and average inclusion size $\left(\mu \mathrm{m}^{2}\right)$. Mean \pm s.d.; $n=3$. Scale bar, $10 \mu \mathrm{m}$. 
a

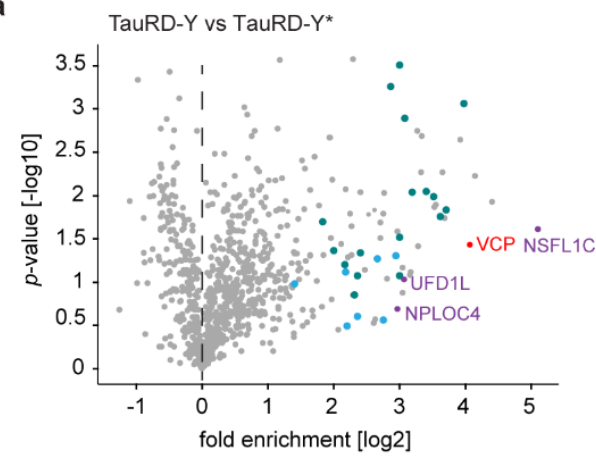

d

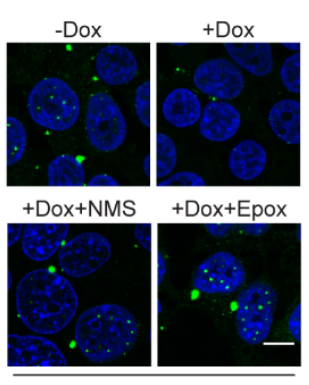

Tet-TauRD-Y* b

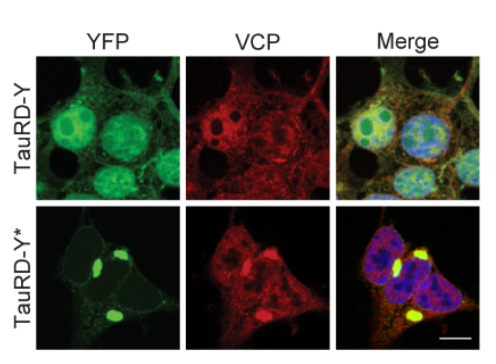

e

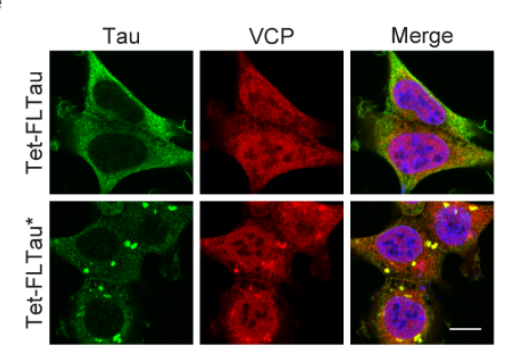

C

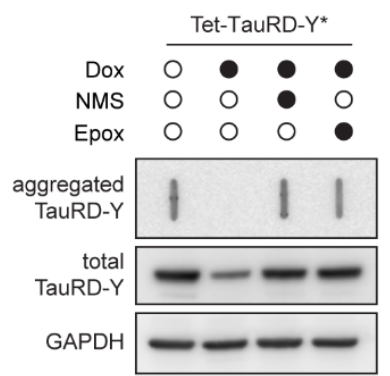

f

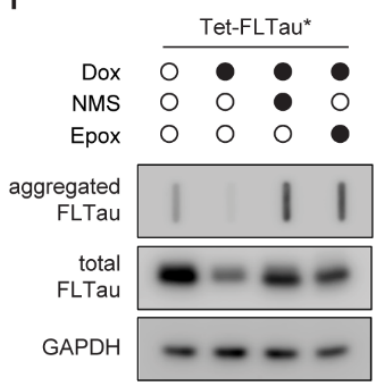


Fig. 2 Disaggregation of Tau aggregates is dependent on VCP activity. a Volcano plot of

384 TauRD-Y interactome from TauRD-Y and TauRD-Y* cells. Unlabeled green and blue symbols

385 represent proteasome subunits of $19 \mathrm{~S}$ and 20S, respectively. VCP and its cofactors are

386 highlighted. b Association of VCP with TauRD-Y inclusions. Immunofluorescence staining of

387 VCP (red) and YFP fluorescence of TauRD-Y (green) in TauRD-Y and TauRD-Y* cells. Scale

388 bar, $10 \mu \mathrm{m}$. c Filter trap analysis of lysates from Tet-TauRD-Y* cells treated for $24 \mathrm{~h}$ with

389 doxycycline (Dox; $50 \mathrm{ng} / \mathrm{mL}$ ) alone or in combination with NMS-873 (NMS; $2.5 \mu \mathrm{M})$ or

390 Epoxomicin (Epox; $50 \mathrm{nM}$ ). Aggregated and total TauRD-Y levels were determined by

391 immunoblotting against GFP. GAPDH served as loading control. d Representative images of

392 Tet-TauRD-Y* cells treated as in (c). Scale bar, $10 \mu \mathrm{m}$. e Association of VCP with FLTau

393 inclusions. Immunofluorescence staining of VCP (red) and Tau with Tau-5 antibody (green) in

394 FLTau and FLTau* cells. Scale bar, $10 \mu \mathrm{m}$. f Filter trap analysis of lysates from Tet-FLTau*

395 cells treated for $24 \mathrm{~h}$ with doxycycline (Dox; $50 \mathrm{ng} / \mathrm{mL}$ ) alone or in combination with NMS-873

396 (NMS; $2.5 \mu \mathrm{M}$ ) or Epoxomicin (Epox; $50 \mathrm{nM}$ ). Aggregated and total FLTau levels were

397 determined by immunoblotting using AT8 and Tau-5 antibodies, respectively. GAPDH served as

398 loading control.

399 


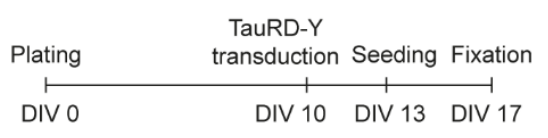

b
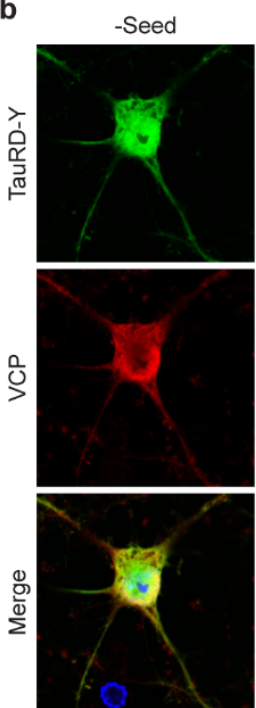

e

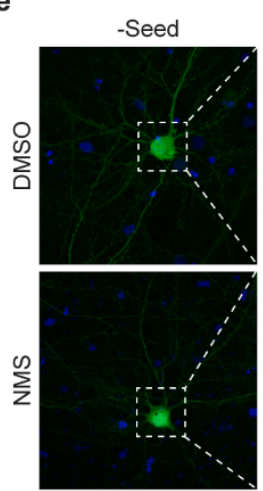

+ Seed
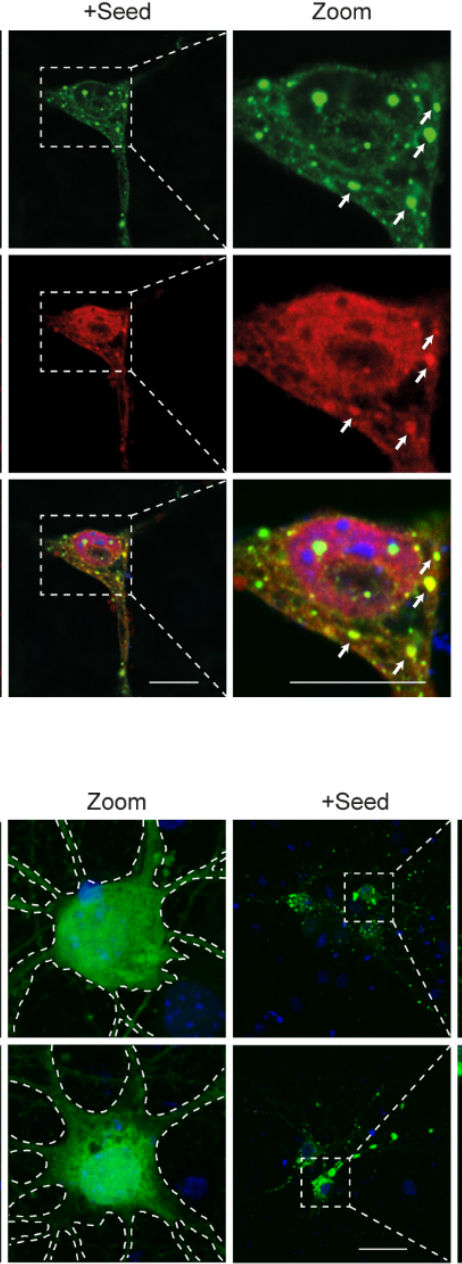

C

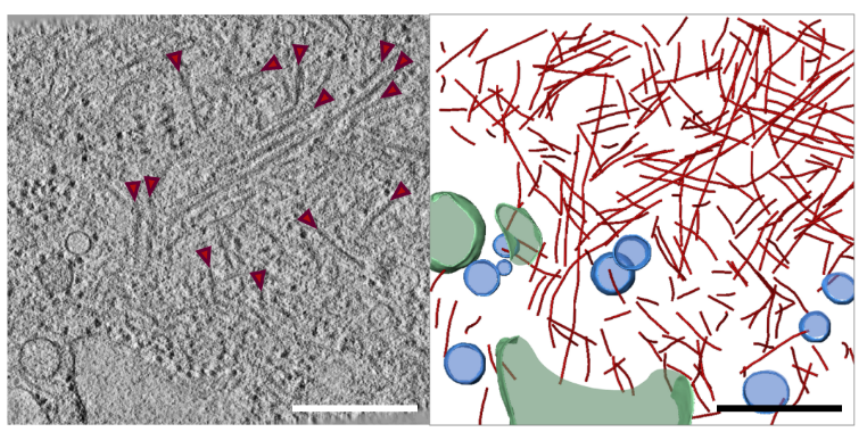

d
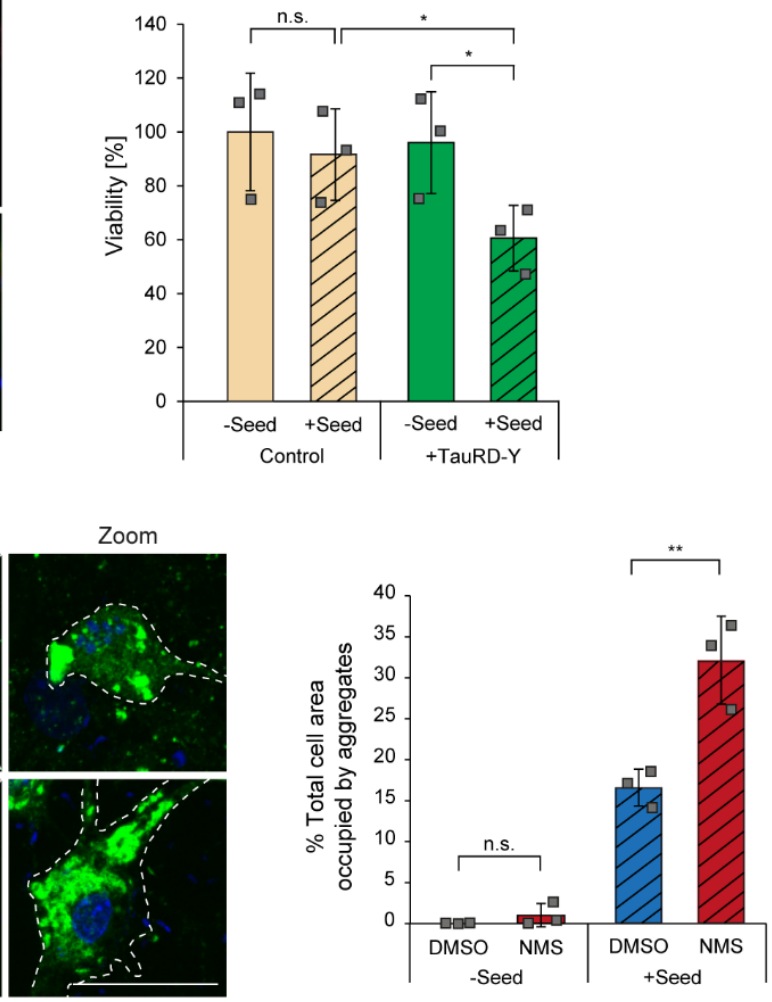

402

Fig. 3 Disaggregation of TauRD-Y aggregates in primary neurons is dependent on VCP

404 activity. a Schematic representation of experimental timeline in primary neurons. DIV, days in

405 vitro. b Association of VCP with TauRD-Y inclusions in primary neurons. Immunofluorescence

406 staining of VCP (red) and YFP fluorescence of TauRD-Y (green). Arrows point to TauRD-Y

407 inclusions containing VCP. Scale bars, $10 \mu \mathrm{m}$. c Fibrillar TauRD-Y aggregates in primary

408 neurons. Left, a $1.4 \mathrm{~nm}$ thick tomographic slice of a TauRD inclusion from neurons is shown. 
409 Red arrows indicate TauRD-Y fibrils. Right, 3D rendering of corresponding tomogram showing 410 TauRD-Y fibrils (red), vesicles (blue) and ER (green). Scale bar, $350 \mathrm{~nm}$. d Toxicity of TauRD411 Y aggregation in primary neurons. Untransduced neurons or neurons transduced with TauRD-Y 412 were treated with cell lysates containing TauRD-Y aggregates for 4 days where indicated.

413 Viability was measured using an MTT assay. Mean \pm s.d.; $n=3 ;{ }^{*}<<0.05$ (Control + Seed vs 414 TauRD-Y + Seed, $\mathrm{p}=0.0184$; TauRD-Y - Seed vs TauRD-Y + Seed, $\mathrm{p}=0.142) ;$ n.s. non415 significant (Control - Seed vs Control + Seed, $\mathrm{p}=0.2074)$ from two-way ANOVA with Tukey 416 post hoc test. e Left, representative images of primary neurons expressing TauRD-Y, exposed to 417 cell lysates containing TauRD-Y aggregates and treated for $4 \mathrm{~h}$ with NMS-873 (NMS; $0.5 \mu \mathrm{M}$ ) 418 where indicated. Scale bars, $20 \mu \mathrm{m}$. Right, quantification of area occupied by TauRD-Y 419 aggregates as a percentage of total area of cells. Mean \pm s.d.; $\mathrm{n}=3 ; * * \mathrm{p}<0.01$ (+ Seed + DMSO vs $420+$ Seed + NMS, $p=0.0098) ;$ n.s. non-significant $(-$ Seed + DMSO vs - Seed + NMS, $p=0.2998)$ 421 from unpaired $t$ test. 
a

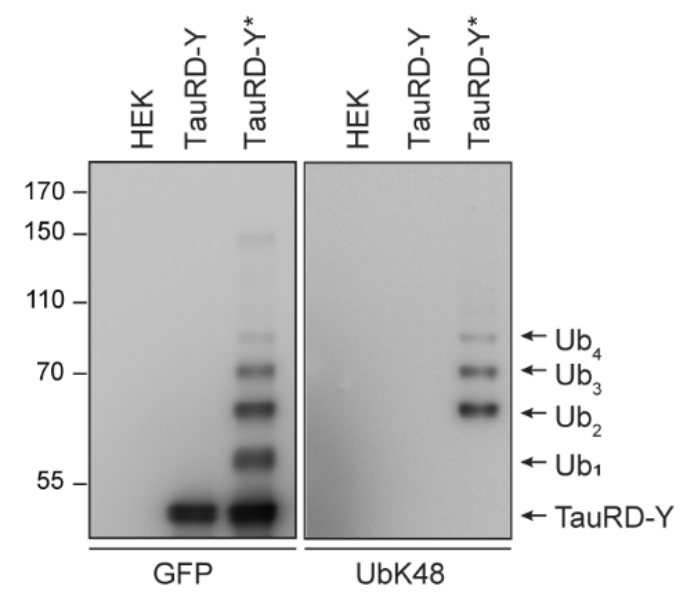

C
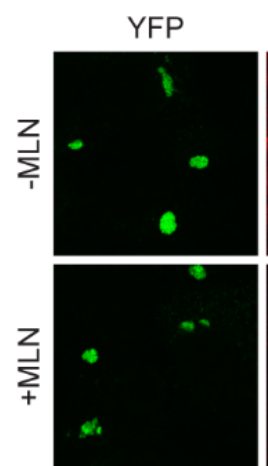
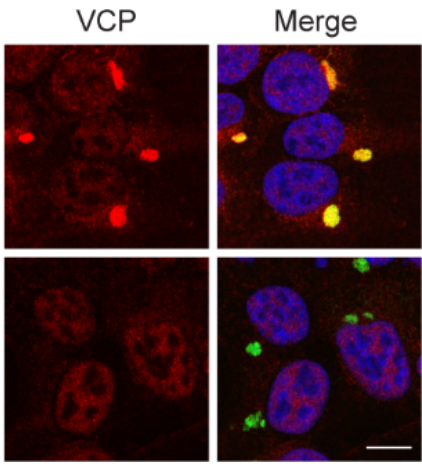

b

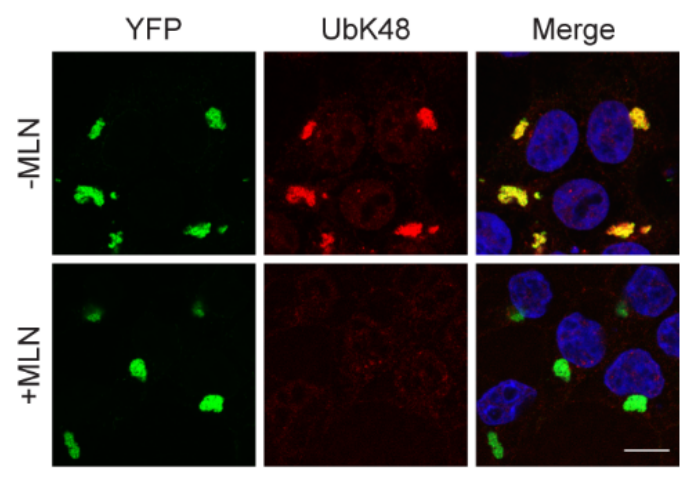

d

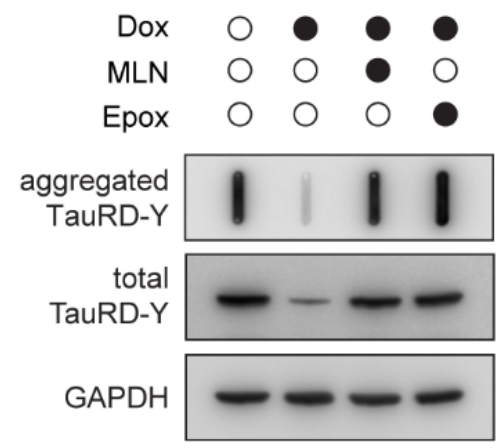

425 Fig. 4 Ubiquitination is necessary for VCP recruitment and disaggregation.

426 a Immunoprecipitation of TauRD-Y from lysates of control HEK cells, TauRD-Y and TauRD-

$427 \mathrm{Y}^{*}$ cells in the presence of $0.1 \%$ SDS using anti-GFP beads. Eluates were analyzed by

428 immunoblotting with antibodies against GFP and K48-linked ubiquitin chains (UbK48). The

429 TauRD-Y band shows the unmodified protein and arrowheads point at increments in ubiquitin

430 conjugation $\left(\mathrm{Ub}_{1}-\mathrm{Ub}_{4}\right)$. $\mathbf{b}$ Inhibition of ubiquitylation of TauRD inclusions. TauRD-Y* cells

431 were treated for $12 \mathrm{~h}$ with MLN7243 (MLN; $0.5 \mu \mathrm{M})$ followed by immunofluorescence analysis

432 with a UbK48 antibody (red). c Inhibition of TauRD ubiquitylation prevents VCP association.

433 TauRD-Y* cells were treated as in (b). VCP (red) was visualized by immunofluorescence. Scale

434 bars: $10 \mu \mathrm{m}$. d Filter trap analysis of lysates from Tet-TauRD-Y* cells treated for 24 h with 50 
$435 \mathrm{ng} / \mathrm{mL}$ doxycycline alone or in combination with $0.2 \mu \mathrm{M}$ MLN7243 or $50 \mathrm{nM}$ Epoxomicin.

436 Aggregated and total TauRD-Y levels were determined by immunoblotting against GFP.

437 GAPDH served as loading control.

438 
a

$\underline{\text { VCP variants }}$

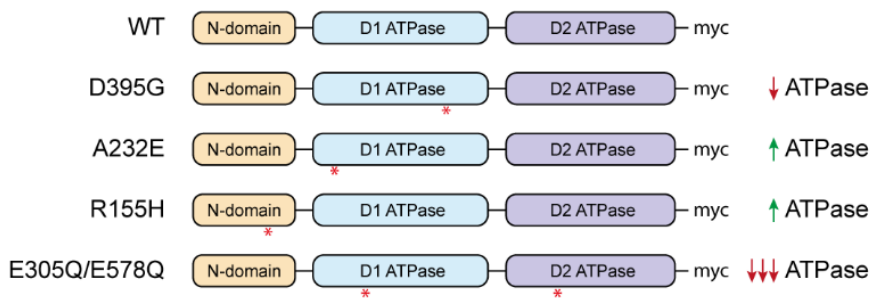

C

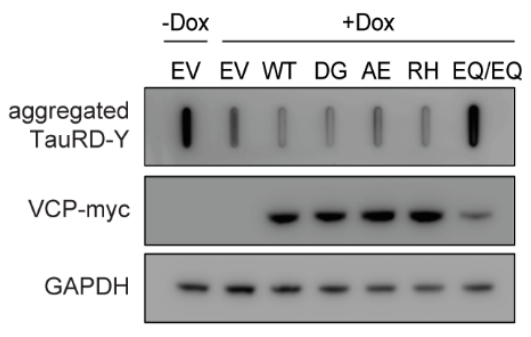

d

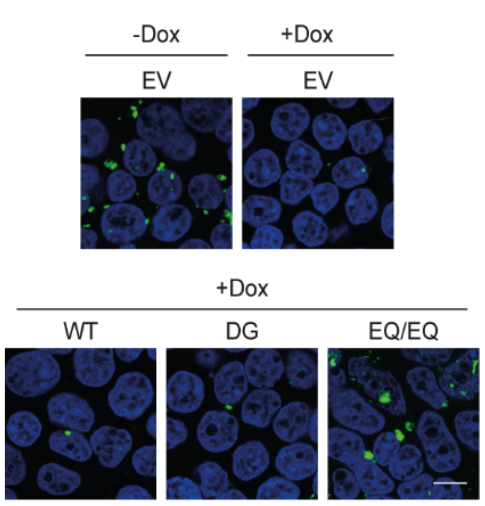

b
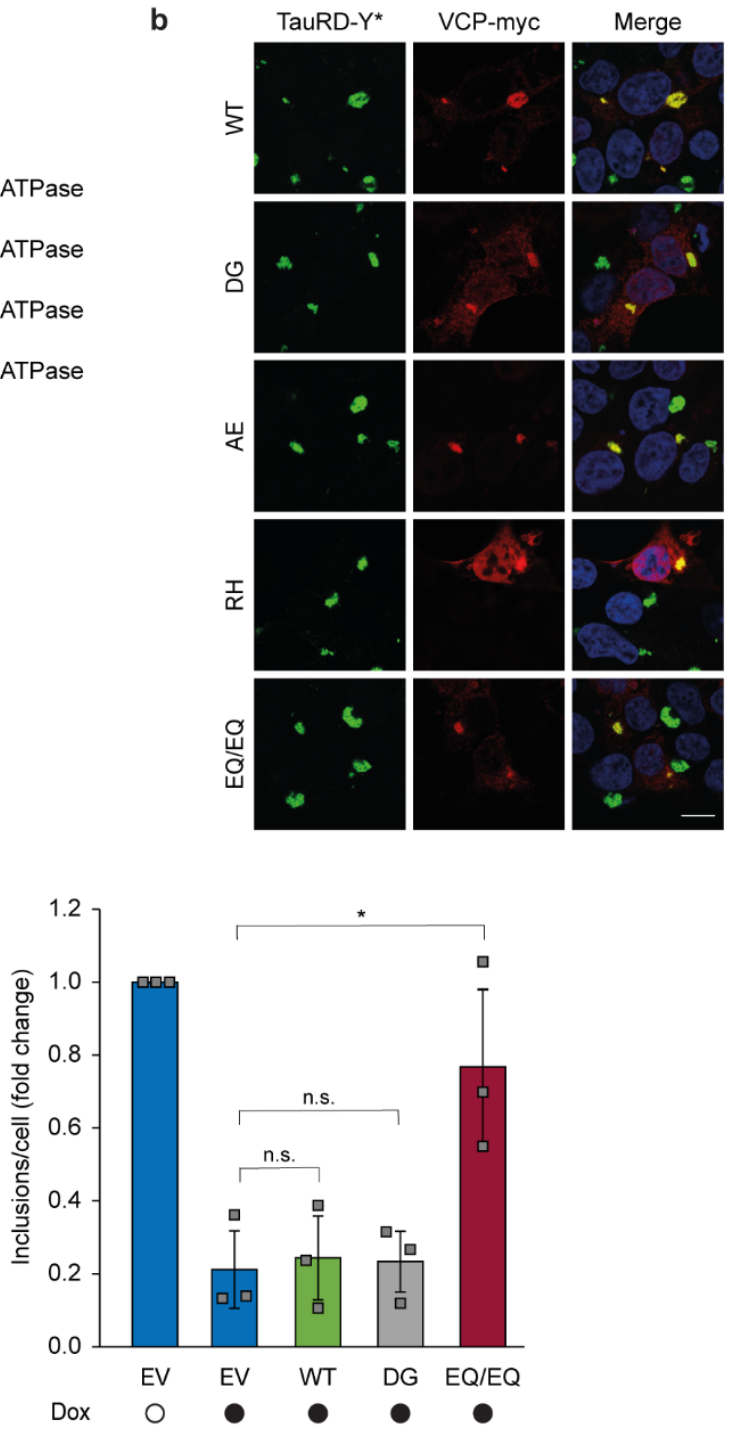

439 Fig. 5 Effects of VCP mutants on Tau disaggregation. a Schematic representation of VCP

440 variants used in this study. Wild type (WT), D395G (DG), A232E (AE), R155H (RH) and

441 E305Q/E578Q (EQ/EQ) VCP were tagged with a C-terminal myc-tag. Red asterisks indicate

442 relative positions of the mutations. b Association of transiently expressed VCP variants with

443 TauRD-Y inclusions. Immunofluorescence staining of myc (red) and YFP fluorescence of

444 TauRD-Y (green) in TauRD-Y* cells. Scale bar, $10 \mu \mathrm{m}$. c Filter trap analysis of lysates from 
445 Tet-TauRD-Y* cells transiently transfected with empty vector (EV) or indicated VCP variants 446 for $24 \mathrm{~h}$, and treated for another $24 \mathrm{~h}$ with doxycycline (Dox; $50 \mathrm{ng} / \mathrm{mL}$ ). Aggregated TauRD-Y 447 and overexpressed VCP levels were determined by immunoblotting against GFP and myc, 448 respectively. GAPDH served as loading control. d Left, representative images of Tet-TauRD-Y* 449 cells treated as in (c). Scale bar, $10 \mu \mathrm{m}$. Right, quantification of aggregate foci in (d). Mean \pm 450 s.d.; $n=3 ;>400$ cells analyzed per experiment; *p<0.05 (EV + Dox vs EQ/EQ + Dox, $p=$ 451 0.0209); n.s. non-significant $(E V+$ Dox vs WT + Dox, $p=0.5017 ; E V+$ Dox vs DG + Dox, $p=$ 452 0.7172) from two-tailed Student's paired t-test. 
a

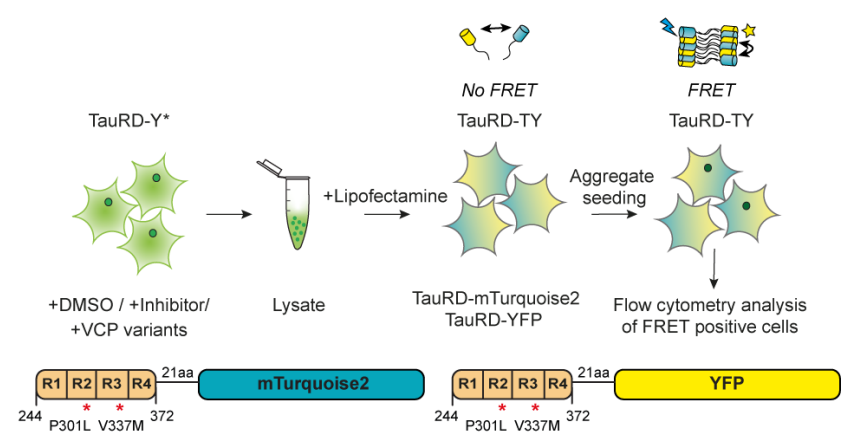

b

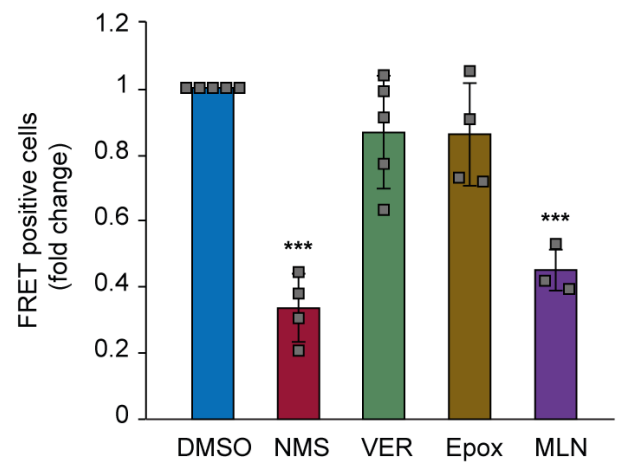

C

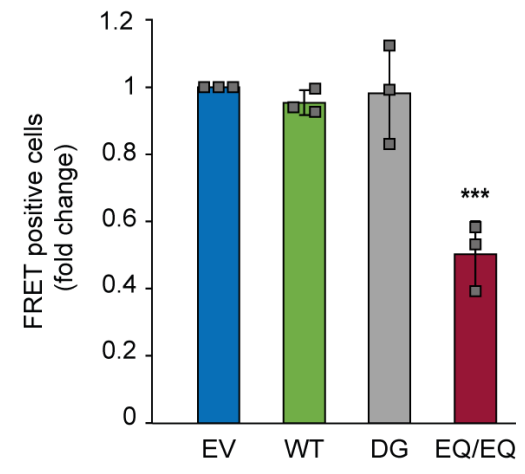

d
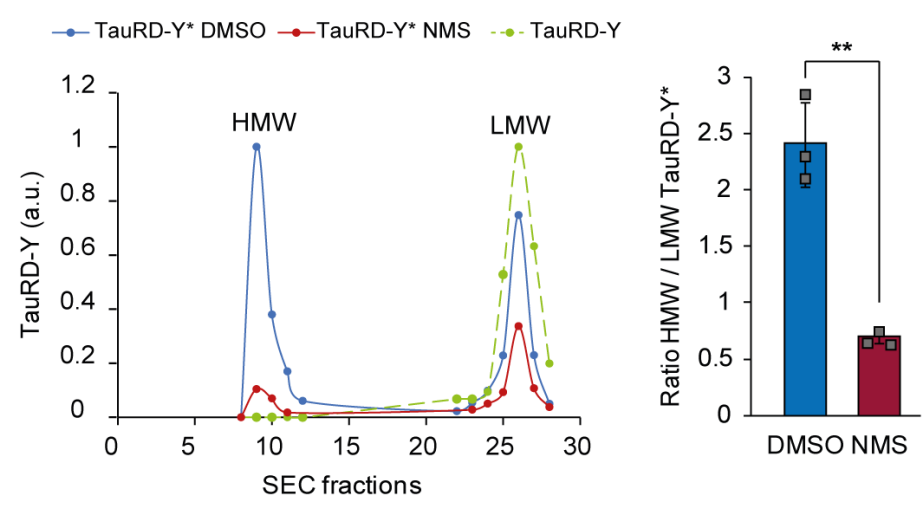

Fig. 6 VCP-mediated disaggregation generates seeding-competent TauRD-Y species.

454 a Experimental scheme to assess the effects of inhibitors of VCP, Hsp70, proteasome and 455 ubiquitylation on the level of TauRD-Y aggregate seeds in TauRD-Y* cells. b Flow cytometry 456 analysis of aggregate seeding in TauRD-TY reporter cells after addition of lysates from TauRD$\mathrm{Y}^{*}$ cells treated with NMS (VCP inhibitor), VER (Hsp70 inhibitor), Epox (proteasome inhibitor) and MLN (ubiquitylation inhibitor). Fold changes with respect to DMSO treated cells are shown.

459 Mean \pm s.d.; NMS and Epox $n=4$, VER $n=5$, MLN $n=3 ; * * * p<0.001$ (DMSO vs NMS, $p=8.69 x$

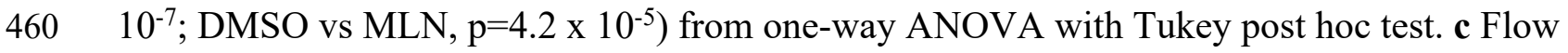
461 cytometry analysis of aggregate seeding in TauRD-TY reporter cells after addition of lysates 462 from TauRD-Y* cells transfected with empty vector (EV), wild-type (WT), D395G (DG) and 
463 ATPase deficient E305Q/E578Q (EQ/EQ) VCP constructs. Fold changes with respect to EV 464 transfected cells are shown. Mean \pm s.d. $n=3 ;{ }^{* * *}<<0.001(E V$ vs $E Q / E Q, p=0.0007)$ from one465 way ANOVA with Tukey post hoc test. $\mathbf{d}$ Left, fractionation of TauRD-Y from DMSO and 466 NMS-873 treated lysates of TauRD-Y* cells by size exclusion chromatography (SEC). Equal 467 amounts of total lysate protein were analyzed. Y-axis represents the relative amount of TauRD-Y 468 in the high molecular weight (HMW) and the low molecular weight (LMW) fractions quantified 469 by immunoblotting. Right, ratio of TauRD-Y in HMW/LMW fractions. Mean \pm s.d.; $\mathrm{n}=3$. $470 \quad * * \mathrm{p}<0.01(\mathrm{p}=0.002)$ from two-tailed Student's paired t-test. 


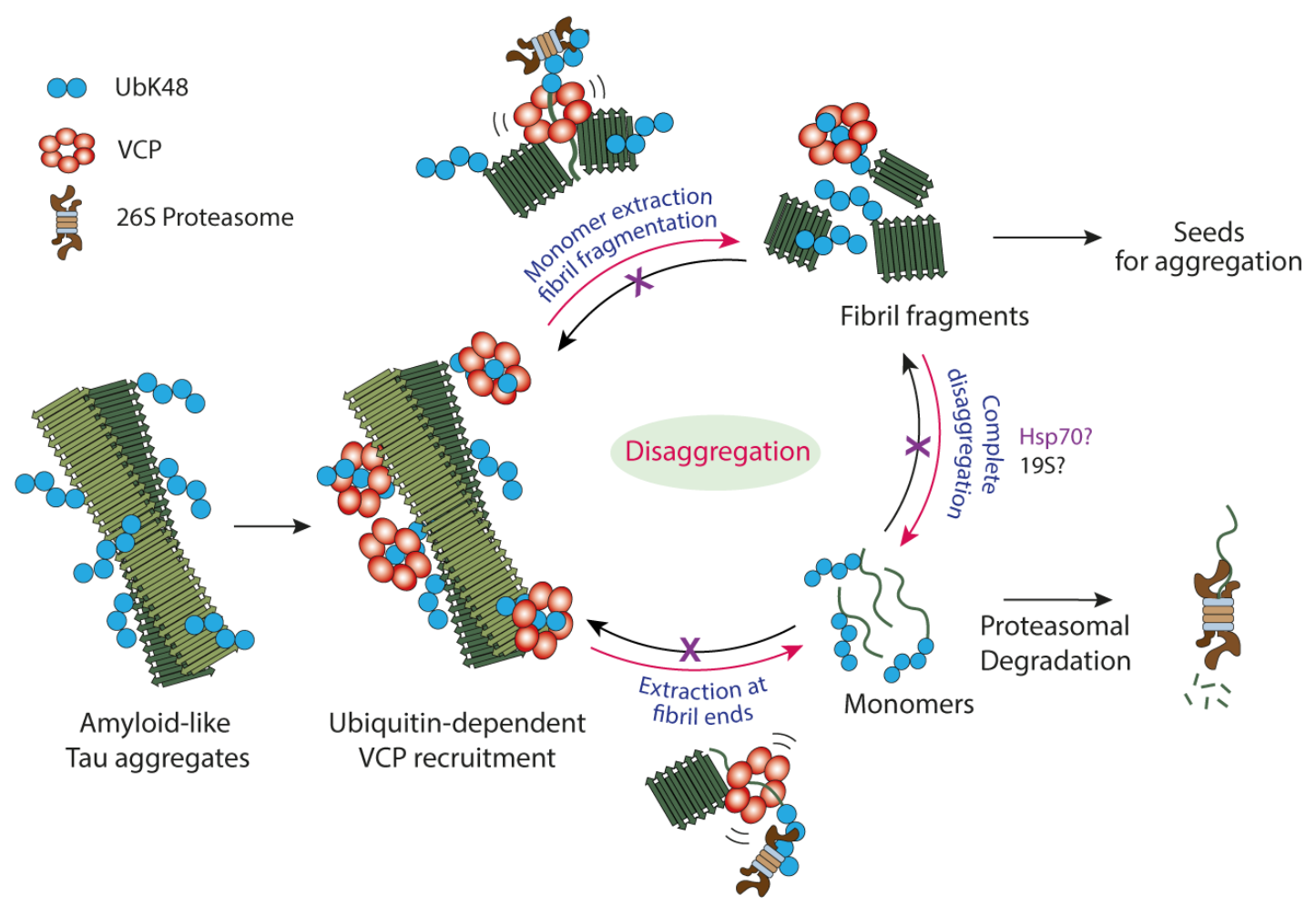

472 Fig. 7 Model of VCP-mediated disaggregation of amyloid-like Tau aggregates. Modification

473 of aggregates with K48-linked ubiquitin chains allows recruitment of VCP. VCP may extract

474 ubiquitylated Tau monomer from fibril ends or from within fibrils. Monomers are directly

475 targeted for proteasomal degradation. Extraction from internal sites results in fibril fragmentation

476 and generation of oligomers that act as seeds for aggregation. Completion of oligomer

477 disaggregation may be accomplished by the 19S proteasome, perhaps with participation of the

478 Hsp70 system (purple). Hsp70 may also contribute to aggregate clearance by preventing re-

479 aggregation of disaggregation products. 
482

483

484

485

486

487

488

489

490

491

492

493

494

495

496

497

498

499

500

501

502

\section{$\underline{\text { Plasmids }}$}

The N1-TauRD (P301L/V337M)-EYFP and N1-FLTau (0N4R, P301L/V337M)-EYFP constructs were previously described ${ }^{37,62}$. To generate TauRD (P301L/V337M) and FLTau (0N4R, P301L/V337M) without fluorescent tag, a stop codon was introduced in the N1-TauRD (P301L/V337M)-EYFP and N1-FLTau (0N4R, P301L/V337M)-EYFP plasmids after the Tau sequence using the Q5 site directed mutagenesis (SDM) kit (New England Biolabs). Tau fragments were subcloned into pcDNA3.1 by restriction digestion and further into pCW57.1MAT2A all-in-one tet-off lentiviral backbone (a gift from David Sabatini (Addgene plasmid \# 100521) $)^{71}$ by Gibson assembly. TauRD (P301L/V337M) construct contains a C-terminal myctag separated from TauRD by a 4 aa (GGSG) linker.

Wild type (WT) VCP (Addgene \#23971), A232E VCP (Addgene \#23973), R155H VCP (Addgene \#23972) and E305Q/E578Q VCP (Addgene \#23974) sequences were derived from plasmids described previously ${ }^{34}$. A C-terminal myc tag and stop codon was introduced using SDM followed by subcloning the VCP-myc fragments into pcDNA3.1. The D395G VCP construct was generated by introducing the D395G mutation in WT-VCP plasmid by SDM. All mutations were verified by sequencing. The plasmid expressing wild type firefly luciferase fused to EGFP (Fluc-GFP) was previously described ${ }^{56}$.

Lentiviral packaging plasmid pVsVg was a gift from Dieter Edbauer. psPAX2 (Addgene \#12260) and pMD2.G (Addgene \#12259) also used for lentiviral production were gifts from Didier Trono. pFhSynW2 TauRD (P301L/V337M)-EYFP used for TauRD-EYFP expression in mouse primary neurons was previously described ${ }^{62}$. 
Cell lines and cell culture

505 Cells expressing constitutive and tet-regulated TauRD-Y (TauRD-Y and Tet-TauRD-Y cell

506 lines, respectively), FRET biosensor TauRD-TY, and FLTau-Y cells were previously

507 described ${ }^{37,62}$. Tet-FLTau and Tet-TauRD cell lines were generated by transducing HEK293T

508 cells with $200 \mu \mathrm{L}$ concentrated lentivirus in presence of $0.8 \mu \mathrm{g} / \mathrm{mL}$ Polybrene (Sigma).

509 Transduced cells were selected with $10 \mu \mathrm{g} / \mathrm{mL}$ Blasticidin (Thermo) and thereafter sorted in 96

510 well-plates with a BD FACS Aria III (BD Biosciences) (Imaging Facility, MPI Biochemistry).

511 Monoclonal cell lines stably expressing FLTau and TauRD were screened by

512 immunofluorescence staining and immunoblotting followed by amplification.

513 All cell lines were cultured in Dulbecco's Modified Eagle Medium (Biochrom)

514 supplemented with 10\% FBS (Gibco), 2 mM L-glutamine (Gibco), 100 units/mL penicillin and

$515100 \mu \mathrm{g} / \mathrm{mL}$ streptomycin (Gibco), and non-essential amino acid cocktail (NEAA) (Gibco) and

516 grown at $37{ }^{\circ} \mathrm{C}$ at $5 \% \mathrm{CO}_{2}$. TauRD-TY and FLTau-Y cells were maintained in presence of

$517200 \mu \mathrm{g} / \mathrm{mL}$ G418 (Gibco). HEK293 cells stably expressing Fluc-GFP were maintained in

518 presence of $50 \mu \mathrm{g} / \mathrm{mL}$ hygromycin (Thermo).

$520 \quad$ Generation of cell lines propagating Tau aggregates

521 Tau aggregation was induced by addition of TauRD aggregates as described previously ${ }^{37}$.

522 Briefly, HEK293 cells expressing TauRD-Y were initially treated with fibrillar aggregates

523 generated in vitro from purified TauRD and clones that displayed the ability to maintain TauRD-

524 Y aggregates for multiple passages were selected. Aggregate-containing TauRD-Y* cells were

525 lysed in Triton buffer $(0.05 \%$ Triton X-100/PBS (Gibco) supplemented with protease inhibitor 526 cocktail (Roche, EDTA-free) and benzonase (prepared in-house)) and kept on ice for $20 \mathrm{~min}$. 
Cell lysate was centrifuged at 1,000 x g for $5 \mathrm{~min}$ and the supernatant was collected. Protein concentration in cell lysates was determined using Bradford assay (Bio-Rad). $30 \mu \mathrm{g}$ of freshly prepared lysate was diluted in $100 \mu \mathrm{L}$ Opti-MEM Reduced Serum Medium (Gibco). In a separate tube $4 \mu \mathrm{L}$ Lipofectamine 2000 was diluted in $100 \mu \mathrm{L}$ Opti-MEM and incubated at room temperature (RT) for $5 \mathrm{~min}$. Contents of the tubes were gently mixed and incubated at RT for 20 min. The lysate-lipofectamine mixture was applied to naïve cells expressing soluble TauRD-Y, plated at 150,000 cells/well in a 12 -well plate. $24 \mathrm{~h}$ later, cells were transferred to a 6 -well plate and 3 days later to $10 \mathrm{~cm}$ dishes ( $<200$ cells per dish) for 8 days, until clearly visible colonies were observed. Colonies were screened for the presence of YFP positive aggregates with an Axio Observer fluorescent microscope (Zeiss). Monoclonal cells displaying aggregate morphology similar to parental cells were amplified and frozen until use. TauRD, FLTau and FLTau-Y expressing cells were similarly seeded with cellular TauRD aggregates ${ }^{37}$ and cultured for several days before experiments were performed with a polyclonal cell population.

\section{Lentivirus production}

For primary neuron transduction: HEK293T cells (LentiX 293T cell line, Takara) for lentiviral packaging were expanded to $70-85 \%$ confluency in DMEM Glutamax $(+4.5 \mathrm{~g} / \mathrm{L}$ D-glucose, pyruvate) supplemented with 10\% FBS (Sigma), 1\% G418 (Gibco), 1\% NEAA (Thermo Fisher) and 1\% HEPES (Biomol). Only low passage cells were used. For lentiviral production, a threelayered $525 \mathrm{~cm}^{2}$ flask (Falcon) was seeded and cells were henceforth cultured in medium without G418. On the following day, cells were transfected with the expression plasmid pFhSynW2 (TauRD-Y), the packaging plasmid psPAX2 and the envelope plasmid pVsVg using TransITLenti transfection reagent (Mirus). The transfection mix was incubated for 20 min at RT. The 
cell medium was exchanged in the meantime. $10 \mathrm{~mL}$ of transfection mix was added to the flask,

551 followed by incubation overnight. The medium was exchanged on the following day. After 48-

$55252 \mathrm{~h}$, culture medium containing the viral particles was collected and centrifuged for $10 \mathrm{~min}$ at

$5531,200 \mathrm{x}$ g. The supernatant was filtered through $0.45 \mu \mathrm{m}$ pore size filters using $50 \mathrm{~mL}$ syringes,

554 and Lenti-X concentrator (Takara) was added. After an overnight incubation at $4{ }^{\circ} \mathrm{C}$, samples

555 were centrifuged at $1,500 \mathrm{x}$ g for $45 \mathrm{~min}$ at $4{ }^{\circ} \mathrm{C}$, the supernatant was removed and the lentivirus

556 pellet was resuspended in $150 \mu \mathrm{L}$ TBS-5 buffer (50 mM Tris- $\mathrm{HCl} \mathrm{pH}$ 7.8, $130 \mathrm{mM} \mathrm{NaCl,} 10$

$557 \mathrm{mM} \mathrm{KCl}, 5 \mathrm{mM} \mathrm{MgCl} 2)$. After aliquoting, lentivirus was stored at $-80{ }^{\circ} \mathrm{C}$.

558 For HEK293T transduction: HEK293T cells (LentiX 293T cell line, Takara) were transfected in

$55910 \mathrm{~cm}$ dishes with packaging plasmid psPAX2, envelope plasmid pMD2.G and expression

560 plasmids (pCW Tet-off FLTau and TauRD) using Lipofectamine 3000. 48 h later virus-

561 containing media was harvested and centrifuged for $5 \mathrm{~min}$ at 1,000 x g. Lenti-X concentrator was

562 added to supernatant, incubated overnight at $4{ }^{\circ} \mathrm{C}$ and the following day centrifuged for $45 \mathrm{~min}$

563 at $1,500 \mathrm{x}$ g at $4{ }^{\circ} \mathrm{C}$. The lentiviral pellet was resuspended in $1 \mathrm{~mL}$ PBS, aliquoted and stored at -

$56480^{\circ} \mathrm{C}$.

565

566 Primary neuronal cultures

567 Primary cortical neurons were prepared from E15.5 CD-1 wild type mouse embryos. All

568 experiments involving mice were performed in accordance with the relevant guidelines and

569 regulations. Pregnant female mice were sacrificed by cervical dislocation. The uterus was

570 removed from the abdominal cavity and placed into a $10 \mathrm{~cm}$ sterile Petri dish on ice containing

571 dissection medium, consisting of Hanks' balanced salt solution (HBSS) supplemented with

0.01 M HEPES, 0.01 $\mathrm{M} \mathrm{MgSO}_{4}$ and 1\% penicillin/streptomycin. Each embryo was isolated, 
573 heads were quickly cut, and brains were removed from the skull and immersed in ice-cold 574 dissection medium. Cortical hemispheres were dissected, and meninges were removed. The 575 cortices were collected in a $15 \mathrm{~mL}$ sterile tube and digested with $0.25 \%$ trypsin containing $1 \mathrm{mM}$ 576 ethylenediaminetetraacetic acid (EDTA) and $15 \mu \mathrm{L} 0.1 \%$ DNAse I for $20 \mathrm{~min}$ at $37^{\circ} \mathrm{C}$. The 577 digestion was stopped by removing the supernatant and washing the tissue twice with 578 Neurobasal medium (Invitrogen) containing 5\% FBS. The tissue was resuspended in $2 \mathrm{~mL}$ 579 Neurobasal medium and triturated to achieve a single cell suspension. Cells were spun at $130 \mathrm{x}$ $580 \mathrm{~g}$, the supernatant was removed, and the cell pellet was resuspended in Neurobasal medium with $5812 \%$ B-27 supplement (Invitrogen), 1\% L-glutamine (Invitrogen) and 1\% penicillin/streptomycin 582 (Invitrogen). For immunofluorescence microscopy, neurons were cultured in 24-well plates on $58313 \mathrm{~mm}$ coverslips coated with $1 \mathrm{mg} / \mathrm{mL}$ poly-D-lysine (Sigma) and $1 \mu \mathrm{g} / \mathrm{mL}$ laminin (Thermo 584 Fisher Scientific) (100,000 neurons per well). For biochemical assays, neurons were cultured in 585 12-well plates coated in the same way (200,000 neurons per well). For viability measurements, 586 neurons were cultured in 96 -well plates coated in the same way $(18,000$ neurons per well).

587 Lentiviral transduction was performed at 10 days in vitro (DIV 10). Virus preparation was 588 thawed and immediately added to freshly prepared neuronal culture medium. Neurons in 24-well 589 plates received $1 \mu \mathrm{L}$ of virus per well. Neurons in 12 -well plates received $1.5 \mu \mathrm{L}$ of virus per 590 well. Neurons in 96-well plates received $0.15 \mu \mathrm{L}$ of virus per well. A fifth of the medium from 591 cultured neurons was removed and the equivalent volume of virus-containing medium was 592 added. Three days after transduction (DIV 13), 2, 6 or $12 \mu \mathrm{g}$ of HEK293 cell lysate containing 593 TauRD-Y aggregates, mixed with fresh medium (one tenth of medium volume in the well), were 594 added to the neuronal cultures in 96, 24 or 12-well plates, respectively. HEK293 cell lysate for 
neurons was prepared by brief sonication of aggregate-containing cells in PBS. Six days after transduction (DIV 16), neurons were treated with inhibitor or DMSO as control.

\section{$\underline{\text { Neuronal viability assay }}$}

Viability of transduced neurons was determined using Thiazolyl Blue Tetrazolium Bromide (MTT; Sigma-Aldrich). Seven days after transduction (DIV 17), cell medium was exchanged for $100 \mu \mathrm{L}$ of fresh medium, followed by addition of $20 \mu \mathrm{L}$ of $5 \mathrm{mg} / \mathrm{ml} \mathrm{MTT} / \mathrm{PBS}$ and incubation for $4 \mathrm{~h}$ at $37^{\circ} \mathrm{C}, 5 \% \mathrm{CO}_{2}$. Subsequently, $100 \mu \mathrm{L}$ solubilizer solution (10\% SDS, $45 \%$ dimethylformamide in water, $\mathrm{pH} 4.5$ ) was added, and on the following day absorbance was measured at $570 \mathrm{~nm}$. Each condition was measured in triplicates per experiment and absorbance values were averaged for each experiment. The individual values for the 'Control-Seed' condition obtained for each of the three experiments were normalized by the mean of these values. The values of all other conditions were normalized by the new value of the 'ControlSeed' condition of the corresponding independent experiment.

\section{$\underline{\text { Plasmid and siRNA transfection }}$}

Plasmids were transfected with Lipofectamine 2000 (Thermo) after manufacturer's instructions in 12- or 6-well plates using 2 or $4 \mu \mathrm{g}$ DNA. All siRNAs were obtained from Dharmacon as ONTARGETplus SMART pools: VCP (L-008727-00-0005), Atg5 (M-004374-04-0005), Atg7 (L020112-00-0005), PSMD11 (L-011367-01-0005), non-targeting control (D-001810-03-20). Cells were plated in 24-well plates in $500 \mu \mathrm{L}$ antibiotic free DMEM. $2 \mu \mathrm{L}$ of Dharmafect transfection reagent and 50-100 $\mathrm{nM}$ of siRNA were diluted each in $50 \mu \mathrm{L}$ Opti-MEM and incubated at RT for 5 min. Contents of the tubes were mixed gently by pipetting and incubated further at RT for 15 
618 min. Subsequently, the transfection mixture was added to the cells drop-wise. $24 \mathrm{~h}$ later cells

619 were split and plated in 12- or 6-well plates and allowed to grow for up to $96 \mathrm{~h}$ before

620 immunoblotting or immunofluorescent staining.

621

$622 \quad$ Antibodies and chemicals

623 The following primary antibodies were used for immunoblotting or immunofluorescent staining:

624 anti-VCP (AbCam \#ab11433), anti-VCP (Novus Biologicals \#NB100-1558) (Fig. 2e and

625 Supplementary Fig. 9a), anti-GFP (Roche \#11814460001), anti-ubiquitin Lys48-specific

626 (Millipore \#05-1307), anti-ubiquitin Lys63-specific (AbCam \#ab179434), anti-ubiquitin (P4D1)

627 (SantaCruz \#sc-8017), anti-Tau (pS356) (GeneTex \#GTX50165), anti-phospho-Tau (S202,

628 T205) (Thermo \#MN1020), anti-NPLOC4 (Sigma \#HPA021560), anti-UFD1L (AbCam

629 \#ab96648), anti-ubiquitin FK2 (Millipore \#04-263), anti-Tau (Tau-5) (Thermo \#MA5-12808),

630 anti-human Tau/Repeat Domain (2B11) (IBL \#JP10237), anti-LC3B (Sigma \#L7543), anti-Atg5

631 (Cell Signalling \#2630S), anti-Atg7 (Cell Signalling \#8558), anti-PSMD11 (Proteintech \#14786-

632 1-AP), anti-myc (in house, 9E10), anti-GAPDH (Millipore \#MAB374), anti-Tubulin (Sigma

633 \#T6199).

634 The following secondary antibodies were used: Cy5-conjugated anti-mouse (Thermo \#A10524),

635 Cy-5 conjugated anti-rabbit (Thermo \#A10523), Alexa Fluor 647 AffiniPure anti-mouse

636 (Jackson ImmunoResearch \#715-605-151), DyLight 488 anti-mouse (Thermo \#SA5-10166),

637 anti-mouse IgG peroxidase conjugate (Sigma \#A4416) or anti-rabbit peroxidase conjugate

638 (Sigma \#A9169), IRDye 680RD anti-mouse (LI-COR \#926-68070), IRDye 800CW anti-rabbit

639 (LI-COR \#926-32211). 
641 methyladenine (Invivogen), bafilomycin A1 (Invivogen), epoxomicin (Cayman Chemical),

642 NMS-873 (Sigma), CB-5083 (Cayman Chemical), VER-155008 (Sigma), MLN7243

643 (Chemietek). Solutions in DMSO were stored at $-20{ }^{\circ} \mathrm{C}$. 3-Methyadenine was dissolved in $\mathrm{H}_{2} \mathrm{O}$

644 after manufacturer's instructions and applied immediately to cells.

645

646 Immunofluorescence staining

647 HEK293 cells were grown on poly-L-lysine (NeuVitro) coated glass coverslips for 24-48 h in 648 12-well plates before any treatment. At the end of the experiment, media was aspirated and cells 649 were directly fixed in 4\% formaldehyde (w/v) (Thermo, Methanol-free) in PBS for 10 min at RT, 650 washed once with PBS and permeabilized in $0.1 \%$ Triton X-100/PBS for 5 min. Samples were 651 blocked using 5\% low-fat dry milk dissolved in $0.1 \%$ Triton X-100/PBS for $1 \mathrm{~h}$ at RT, followed 652 by incubation with primary antibodies in blocking solution and fluorescently labelled secondary 653 antibodies in PBS. Nuclei were counterstained with DAPI. For amyloid staining, after fixation 654 and permeabilization, cells were incubated with Amylo-Glo (Biosensis TR-300-AG) at a dilution 655 of 1:200/PBS with gentle shaking followed by washing twice with PBS. Cells were not 656 counterstained with DAPI. Coverslips were mounted in fluorescent mounting medium (Dako) on 657 glass slides and stored at $4{ }^{\circ} \mathrm{C}$ until imaging.

Primary neurons: Primary neurons were fixed at DIV 17 with 4\% paraformaldehyde

659 (Santa Cruz) (PFA)/PBS for 15 min; remaining free aldehyde groups of PFA were blocked with $66050 \mathrm{mM}$ ammonium chloride/PBS for $10 \mathrm{~min}$ at RT. Cells were rinsed once with PBS and 661 permeabilized with $0.25 \%$ Triton X-100/PBS for 5 min. After washing with PBS, blocking 662 solution consisting of 2\% BSA (w/v) (Roth) and 4\% donkey serum (v/v) (Jackson 
663 ImmunoResearch Laboratories) in PBS was added for $30 \mathrm{~min}$ at RT. Coverslips were transferred

664 to a light protected humid chamber and incubated with primary antibodies diluted in blocking

665 solution for $1 \mathrm{~h}$. Cells were washed with PBS and incubated with secondary antibody diluted in

666 blocking solution for 30 min and counterstained with DAPI. Coverslips were mounted using

667 Prolong Glass fluorescence mounting medium (Invitrogen).

668

669 Image acquisition (Microscopy)

670 Images were acquired with a Zeiss LSM 780, Leica SP8 FALCON (Imaging Facility, MPI

671 Biochemistry) or a Leica TCS SP8 Laser-scanning confocal microscope (Imaging Facility, MPI

672 Neurobiology) and analyzed using FIJI/ImageJ software. For multifluorescent imaging, samples

673 stained with individual fluorophores were used to correct emission bandwidths and exposure

674 settings to minimize spectral crossover.

675

676 Quantification of aggregates/cell and average size

677 Confocal z-stacks were used to create a maximum intensity projection (MIP) using the image

678 acquisition software ZEN (Zeiss). MIPs were further segmented to define aggregate foci by

679 thresholding. Aggregate number and size were computed by the Analyze Particle function (Size:

680 0-infinity). Cell numbers were determined by counting DAPI stained nuclei with the Cell

681 Counter plugin. Experiments were performed at least 3 times in biologically independent repeats.

682 For neuronal aggregates, neuronal cytoplasm area was calculated by manually selecting a region

683 of interest (ROI) around the soma of the neuron and utilizing the Analyze feature. Aggregate foci

684 were identified by thresholding the MIP images and aggregate size (area), within the previously

685 selected ROI, was calculated by the Analyze Particle function. The percentage of total neuron 
686

687

688

689

690

691

692

693

694

695

696

697

698

699

700

701

702

703

704

705

706

707

708

area occupied by aggregate was the quotient of the division between aggregate area and neuronal cytoplasmic area: (Aggregate area)/(Cytoplasm area) x 100. 60 individual neurons were imaged per condition, in 3 biologically independent replicates.

$\underline{\text { mRNA quantification }}$

Total RNA was isolated using the RNeasy Mini Kit (Qiagen) and reverse transcribed with iScript $^{\mathrm{TM}}$ cDNA Synthesis Kit (Biorad) according to manufacturers' instructions. Quantitative real-time PCR was performed with PowerUp ${ }^{\mathrm{TM}}$ SYBR ${ }^{\mathrm{TM}}$ Green Master Mix (Applied Biosystems) with a StepOnePlus Real-Time PCR System (Applied Biosystems). CT values were measured and fold changes calculated by the $\Delta \Delta \mathrm{C}(\mathrm{T})$ method $^{72}$ using the RPS18 gene as reference. The following primers were used: RPS18 forward 5'-

TGTGGTGTTGAGGAAAGCA-3' and reverse 5'- CTTCAGTCGCTCCAGGTCTT-3'; Tau forward: 5'-AGCAACGTCCAGTCCAAGTG-3' and reverse: 5'-

CCTTGCTCAGGTCAACTGGT-3'.

Correlative light and electron microscopy (CLEM), cryo-focused ion beam (FIB) and cryo-

\section{Electron Tomography}

$2 \times 10^{4}$ TauRD-Y* cells or $1 \times 10^{5}$ neurons were seeded on EM grids (R2/1, Au 200 mesh grid, Quantifoil Micro Tools) in a $35 \mathrm{~mm}$ dish or 24-well plate and cultured for $24 \mathrm{~h}$ or transduced with lentivirus and treated with aggregate-containing cell lysate as described earlier in section 'Primary neuronal cultures'. The grids were blotted for $10 \mathrm{~s}$ using filter paper and vitrified by plunge freezing into a liquid ethane/propane mixture with a manual plunger. CLEM, cryo-FIB and tomographic data collection were performed as described in detail before ${ }^{73}$. In brief, EM 
grids were mounted onto modified Autogrid sample carriers ${ }^{74}$ and then transferred onto the cryostage of a CorrSight microscope (FEI) for cryo-light microscopy. Images of the samples and TauRD-Y signal were acquired with MAPS software (FEI) in transmitted light and confocal mode using a $5 \mathrm{x}$ and $20 \mathrm{x}$ lens, respectively. The samples were then transferred into a dual-beam (FIB/SEM) microscope (Quanta 3D FEG, FEI) using a cryo-transfer system (PP3000T, Quorum). Cryo-light microscope and SEM images were correlated with MAPS software. Lamellas (final thickness, $100-200 \mathrm{~nm}$ ) were prepared using a $\mathrm{Ga}^{2+}$ ion beam at $30 \mathrm{kV}$ in the regions of the TauRD-Y fluorescence signal. In case of TauRD-Y* cells, an additional layer of platinum was sputter-coated $(10 \mathrm{~mA}, 5 \mathrm{~s})$ on the grids to improve conductivity of the lamellas. The grids were then transferred to a Titan Krios transmission electron microscope (FEI) for tomographic data collection. For the whole procedure, samples were kept at a constant temperature of $-180^{\circ} \mathrm{C}$. Tomographic tilt series were recorded with a Gatan K2 Summit direct detector in counting mode. A GIF-quantum energy filter was used with a slit width of $20 \mathrm{eV}$ to remove inelastically scattered electrons. Tilt series were collected from $-50^{\circ}$ to $+70^{\circ}$ with an increment of $2^{\circ}$ and total dose of $110 \mathrm{e}^{-} / \AA^{2}$ using SerialEM software ${ }^{75}$ at a nominal magnification of 33,000x, resulting in a pixel size of $4.21 \AA$ for TauRD-Y* cells and at a nominal magnification of $42,000 \mathrm{x}$, resulting in a pixel size of $3.52 \AA$ for the primary neurons. In case of TauRD-Y* cells, a Volta phase plate was used together with a defocus of $-0.5 \mu \mathrm{m}$ for contrast improvement $^{76}$.

For image processing of TauRD-Y* cell tomograms, frames were aligned during data collection using in-house software K2align based on previous work ${ }^{77}$ or in case of the primary neuron tomograms by using the software Morioncor2 and Tomoman (https://github.com/williamnwan/TOMOMAN). The IMOD software package ${ }^{78}$ was used for 
tomogram reconstruction. The tilt series were first aligned using fiducial-less patch tracking, and tomograms were then reconstructed by weighted back projection of the resulting aligned images. For segmentation, tomograms were rescaled with a binning factor of four and in case of the primary neurons tomograms filtered with a deconvolution filter (https://github.com/dtegunov/tom_deconv). Tau filaments were traced with XTracing Module in Amira using a short cylinder as a template ${ }^{79}$. The membranes were first segmented automatically with TomoSegMemTV ${ }^{80}$ using tensor voting, and then manually optimized in Amira.

\section{Immunoblotting}

Cells were lysed in RIPA lysis and extraction buffer (Thermo) supplemented with protease inhibitor cocktail and benzonase for 30 min on ice with intermittent vortexing. Protein concentration in total cell lysates was determined using Bradford assay (Bio-Rad) and normalized in all samples before adding 2x SDS sample buffer. Samples were denatured by boiling at $95{ }^{\circ} \mathrm{C}$ for $5 \mathrm{~min}$. Proteins were resolved on NuPAGE 4-12\% gradient gels (Thermo) with MES or MOPS (Thermo) running buffer at $200 \mathrm{~V}$ for $45 \mathrm{~min}$. Proteins were transferred to nitrocellulose or PVDF membranes (Roche) in tris-glycine buffer at $110 \mathrm{~V}$ for $1 \mathrm{~h}$. Membranes were washed once in TBS-T and blocked in 5\% low-fat dry milk dissolved in TBS-T for $1 \mathrm{~h}$ at RT. Subsequently, blots were washed 3 times with TBS-T and probed with primary and secondary antibodies. Chemiluminescence was developed using HRP substrate (Luminata Classico, Merk) and detected on a LAS 4000 (Fuji) or ImageQuant800 (Amersham) imager. AIDA image software (Elysia Raytest) was used to quantify intensity of protein bands. 
Interactome analysis by mass spectrometry

755

756

757

758

759

760

761

762

763

764

765

766

767

768

769

770

771

772

773

774

775

776
SILAC labelling of cells and TauRD-Y immunoprecipitation: Interactome analyses were performed using a stable isotope labelling by amino acids in cell culture (SILAC)-based quantitative proteomics approach ${ }^{81}$. Frozen TauRD-Y and TauRD-Y* cells were thawed in arginine lysine deficient SILAC media (PAA) containing light (L) (Argo, $\mathrm{Lys}_{0}$, Sigma) and heavy $(\mathrm{H})\left(\mathrm{Arg}_{10}, \mathrm{Lys}\right.$, Silantes) amino acid isotopes, respectively, and supplemented with 10\% dialyzed FCS (PAA), 2 mM L-glutamine (Gibco), 100 units/mL penicillin and $100 \mu \mathrm{g} / \mathrm{mL}$ streptomycin (Gibco), and non-essential amino acid cocktail (Gibco). A third cell line, not part of this study but included in the PRIDE entry PXD023400, was simultaneously expanded in SILAC medium supplemented with medium (M) ( $\operatorname{Arg}_{6}, \mathrm{Lys}_{4}$, Silantes) amino acid isotopes, and was processed and analyzed together with TauRD-Y and TauRD-Y* samples. Cells were passaged for a minimum of two weeks to allow efficient incorporation of amino acid isotopes into the cellular proteome. Cells from a $10 \mathrm{~cm}$ dish were washed in PBS, lysed by gentle pipetting in $400 \mu \mathrm{L}$ ice cold lysis buffer (1\% Triton X-100/PBS supplemented with protease inhibitor cocktail and benzonase). Lysates were sonicated briefly and centrifuged at 2,000 x g for $5 \mathrm{~min}$ at $4{ }^{\circ} \mathrm{C} .300 \mu \mathrm{L}$ of the supernatant was removed and protein concentration was determined using Bradford assay (Bio-Rad). $50 \mu \mathrm{L}$ anti-GFP beads ( $\mu$ MACS GFP Isolation kit, Miltenyi Biotech) were added to $500 \mu \mathrm{g}$ total protein diluted in a total volume of $800 \mu \mathrm{L}$ lysis buffer. Lysates were incubated for $1 \mathrm{~h}$ at $4{ }^{\circ} \mathrm{C}$ with end over end rotation at $10 \mathrm{rpm} . \mu$-Columns (Miltenyi Biotech) were placed in the magnetic field of a $\mu$ MACS Separator (Miltenyi Biotech) and equilibrated with $250 \mu \mathrm{L}$ lysis buffer before lysates were applied. Columns were washed 4 times with $1 \mathrm{~mL}$ cold Triton buffer and 2 times with $1 \mathrm{~mL}$ PBS followed by elution in $70 \mu \mathrm{L}$ preheated 1x SDS sample buffer without bromophenol blue. 
MS sample processing: $20 \mu \mathrm{L}$ sample from each of the $\mathrm{H}, \mathrm{M}$ and $\mathrm{L}$ eluates was mixed

778

779

780

781

782

783

784

785

786

787

788

789

790

791

792

793

794

795

796

797

798

799

and processed by the filter-aided sample preparation (FASP) method as previously described ${ }^{82}$.

Samples were loaded in a $30 \mathrm{kDa}$ centrifugation device and washed 3 times with $200 \mu \mathrm{L}$ freshly

prepared urea buffer (UB) (8 M urea, 0.1 M Tris $\mathrm{pH} 8.5)$. Reduction and alkylation was

performed sequentially using $10 \mathrm{mM}$ DTT and $50 \mathrm{mM}$ iodoacetamide in UB, respectively.

Samples were washed 2 times with $200 \mu \mathrm{L} 50 \mathrm{mM}$ ammonium bicarbonate $\left(\mathrm{NH}_{4} \mathrm{HCO}_{3}\right)$ to

remove urea before an over-night trypsin treatment. Peptides were recovered in $40 \mu \mathrm{L}$

$\mathrm{NH}_{4} \mathrm{HCO}_{3}$, acidified with $12 \mu \mathrm{L}$ of a $25 \%$ TFA solution and dried in a vacuum concentrator. The peptides were further fractionated using home-made SAX columns in $200 \mu \mathrm{L}$ microtips by stacking 2 punch-outs of Empore High Performance Extraction Disk (Anion-SR) material. Peptides were sequentially eluted with 6 different Britton \& Robinson buffers (BURB) of decreasing $\mathrm{pH}(\mathrm{pH} 11,8,6,5,4,3)$ and acidified to $1 \%$ TFA. The last elution step was with $\mathrm{MeOH} /$ water (1:1)/1\% formic acid. The fractionated peptides were desalted with home-made micro-columns containing C18 Empore disks and eluted with 70\% ACN 1\% formic acid followed by drying in a vacuum concentrator. The samples were stored at $-20{ }^{\circ} \mathrm{C}$ until analysis. $L C-M S$ : The desalted peptides were dissolved in $5 \mu \mathrm{L}$ of $5 \%$ formic acid, sonicated in an ultrasonic bath, centrifuged and transferred to MS autosampler vials. Samples were analyzed on an Easy nLC-1000 nanoHPLC system (Thermo) coupled to a Q-Exactive Orbitrap mass spectrometer (Thermo). Peptides were separated on home-made spray-columns (ID $75 \mu \mathrm{m}, 20$ cm long, $8 \mu \mathrm{m}$ tip opening, NewObjective) packed with $1.9 \mu \mathrm{m}$ C18 particles (Reprosil-Pur C18AQ, Dr Maisch $\mathrm{GmbH})$ using a stepwise 115 min gradient between buffer A $(0.2 \%$ formic acid in water) and buffer B ( $0.2 \%$ formic acid in acetonitrile). Samples were loaded on the column by the nanoHPLC autosampler at a flow rate of $0.5 \mu \mathrm{L}$ per min. No trap column was used. The 
HPLC flow rate was set to $0.25 \mu \mathrm{L}$ per min during analysis. MS/MS analysis was performed with standard settings using cycles of 1 high resolution (70000 FWHM setting) MS scan followed by MS/MS scans (resolution 17500 FWHM setting) of the 10 most intense ions with charge states of 2 or higher.

MS data analysis: Protein identification and SILAC based quantitation was performed using MaxQuant (version 1.5.4.1) using default settings. The human sequences of UNIPROT (version 2019-03-12) were used as database for protein identification. MaxQuant used a decoy version of the specified UNIPROT database to adjust the false discovery rates for proteins and peptides below 1\%. We used normalized MaxQuant ratios for enrichment analyses to correct for uneven total protein amounts in the SILAC-labeling states. Proteins quantified in at least 2 experiments with normalized $\mathrm{H} / \mathrm{L}$ ratios $\geq 2$ were considered as interactors of TauRD-Y in TauRD-Y* cells. Volcano plot was generated using Perseus1.6.2.3.

\section{Biochemical detection of aggregated Tau}

Cells were lysed for $30 \mathrm{~min}$ on ice in lysis buffer followed by brief sonication or $1 \mathrm{~h}$ in RIPA buffer. Lysates were centrifuged at 2,000 or 1,000 x g for $5 \mathrm{~min}$. The supernatant was carefully removed and protein concentration was normalized across all samples. Lysates were then used for solubility or filter trap assays. Lysates were centrifuged at $186,000 \mathrm{x} \mathrm{g}$ for $1 \mathrm{~h}$ at $4{ }^{\circ} \mathrm{C}$.

Supernatant was removed and the pellet was washed with $200 \mu \mathrm{L}$ PBS and centrifuged again for 30 min. Pellets were disintegrated in PBS by pipetting and boiled in 1x SDS sample buffer. Filter trap assays were performed with $200 \mu \mathrm{g}$ total protein diluted in $200 \mu \mathrm{L}$ lysis buffer. A cellulose acetate membrane $(0.2 \mu \mathrm{m}$ pore size, GE Healthcare) was pre-equilibrated in $0.1 \%$ SDS and affixed to the filter trap apparatus (PR648 Slot Blot Blotting Manifold, Hoefer). Samples were 
823 loaded and allowed to completely pass through the filter under vacuum. Wells were washed 3

824 times with $200 \mu \mathrm{L} 0.1 \% \mathrm{SDS} / \mathrm{H}_{2} \mathrm{O}$ followed by standard immunoblotting of the membrane.

825

826 Detection of Tau ubiquitylation

827 Cells were lysed as described in section Immunoblotting, with the addition of $20 \mathrm{mM} \mathrm{N}$ -

828 ethylmalemide followed by brief sonication and centrifugation at 2,000 x g for 5 min. Protein

829 concentration was determined using Bradford assay (Bio-Rad). $50 \mu \mathrm{L}$ anti-GFP beads were

830 added to $1 \mathrm{mg}$ total protein diluted in a total volume of $600 \mu \mathrm{L}$ RIPA buffer. Lysates were

831 incubated for $1 \mathrm{~h}$ at $4{ }^{\circ} \mathrm{C}$ with end over end rotation. Cell lysates were applied to $\mu$-columns

832 equilibrated with $250 \mu \mathrm{L}$ RIPA buffer. Columns were washed 4 times with $1 \mathrm{~mL} 0.1 \%$

$833 \mathrm{SDS} / \mathrm{PBS}$. Bound proteins were eluted by applying $50 \mu \mathrm{L}$ pre-heated $\left(95^{\circ} \mathrm{C}\right) 1 \mathrm{x}$ SDS sample

834 buffer. Input and eluates were resolved on NuPAGE 4-12\% gradient gels in MOPS running

835 buffer and transferred to nitrocellulose membranes. Membranes were probed with antibodies 836 against GFP or ubiquitin-K48.

837

$838 \quad$ Native-PAGE analysis

839 Tet-TauRD-Y* cells were plated in 12-well plates and transfected with VCP variants using 840 Lipofectamine 2000 for 2 days. Cells were then lysed in $50 \mu \mathrm{L} 0.5 \%$ TritonX-100/PBS

841 supplemented with protease inhibitor cocktail and benzonase for $1 \mathrm{~h}$ on ice. Lysates were 842 centrifuged at $10,000 \mathrm{x}$ g for $2 \mathrm{~min}$ and supernatant was collected. Protein concentration in the 843 supernatant was determined using Bradford assay and normalized in all samples before adding $8442 x$ native sample buffer (40\% glycerol, $240 \mathrm{mM}$ Tris $\mathrm{pH}$ 6.8, $0.04 \%$ bromophenol blue).

845 Samples were analyzed on Novex Value 4 to 12\% Tris-glycine gels (Thermo) using $20 \mathrm{mM}$ Tris 
$846200 \mathrm{mM}$ Glycine buffer at $\mathrm{pH}$ 8.4. Proteins were transferred to nitrocellulose membrane in Tris-

847 glycine buffer, blocked in 5\% low-fat dry milk and co-incubated with primary followed by

848 fluorescent secondary antibodies. Fluorescent signal was detected on an Odyssey Fc imager (LI-

849 COR).

850

$851 \quad$ TauRD-Y seeding assay

852 TauRD-Y* cells were treated with $2 \mu \mathrm{M}$ NMS, $10 \mu \mathrm{M}$ VER or $50 \mathrm{nM}$ epoxomicin for $24 \mathrm{~h}$ or $853 \quad 0.5 \mu \mathrm{M}$ MLN for $12 \mathrm{~h}$, or with DMSO as control and lysed on ice in Triton buffer supplemented 854 with protease inhibitor cocktail and benzonase for $20 \mathrm{~min}$. The amount of TauRD-Y across the 855 samples was normalized by quantifying TauRD-Y by immunoblotting using anti-GFP antibody 856 and anti-GAPDH antibody as loading control. Lysates containing equal amounts of TauRD-Y 857 were combined with Opti-MEM and Lipofectamine 3000, incubated for $20 \mathrm{~min}$ at RT and added 858 to FRET biosensor cells. 24 h later, cells were harvested with trypsin, washed with PBS and 859 analyzed on an Attune NxT flow cytometer (Imaging Facility, MPI Biochemistry). mTurquoise2 860 and FRET fluorescence signals were measured by exciting cells with a $405 \mathrm{~nm}$ laser and 861 collecting fluorescent signal with $440 / 50$ and 530/30 filters, respectively. To measure the YFP 862 fluorescence signal, cells were excited with a $488 \mathrm{~nm}$ laser and emission was collected with a $863530 / 30$ filter. For each sample 50,000 single cells were evaluated. Data was processed using 864 FlowJo v10.7.1 software (FlowJo LLC). After gating single cells, an additional gate was 865 introduced to exclude cells that generate a false-positive signal in the FRET channel due to 866 excitation at $405 \mathrm{~nm}^{83}$. A FRET positive gate was drawn by plotting the FRET fluorescence 867 signal versus the mTurquoise2 fluorescence signal using unseeded cells as reference. 868 
869

870

871

872

873

874

875

876

877

878

879

880

881

882

883

884

885

886

887

888

889

890

$\underline{\text { Size exclusion chromatography of cell lysates }}$

TauRD-Y* cells that had been treated for $24 \mathrm{~h}$ with DMSO or $2 \mu \mathrm{M}$ NMS were analyzed.

Untreated TauRD-Y cells were analyzed as control. Cells were lysed as described in the section

Seeding assay. Lysates were clarified by centrifugation at $1,000 \mathrm{xg}$ for $5 \mathrm{~min}$ at $4{ }^{\circ} \mathrm{C}$ and filtered with a PVDF $0.22 \mu \mathrm{m}$ filter (Millex). The total protein amount of the lysates was determined by

Bradford assay (Bio-Rad). $3 \mathrm{mg}$ total protein was loaded on a Superose 6 HR10/30 (GE

Healthcare) column equilibrated with PBS. The individual fractions separated by size exclusion chromatography were analyzed and quantified by immunoblotting using anti-GFP antibody.

TauRD-Y species were detected in the void volume (HMW) and low molecular weight (LMW)

fractions. Corresponding fractions were pooled and analyzed by immunoblotting using anti-GFP antibody. Seeding experiments were performed as described above, using $0.5 \mathrm{ng}$ TauRD-Y from HMW and LMW fractions.

\section{$\underline{\text { Statistical analysis }}$}

Statistical analysis was performed in Excel, Origin 2019b or GraphPad Prism 7 on data acquired from at least three independent experiments. Matched samples were compared using two-tailed Student's paired t-test. For multiple comparisons, one-way ANOVA followed by a Tukey post hoc test was used.

\section{Data availability}

All data supporting the findings of this study are included in the manuscript and the Supplemental Information, additional data that support the findings of this study are available from the corresponding author upon reasonable request. The mass spectrometry proteomics data associated to Fig. 2a have been deposited to the ProteomeXchange Consortium via the PRIDE ${ }^{84}$ 
891 partner repository (https://www.ebi.ac.uk/pride/archive/) with the dataset identifier PXD023400.

892 This PRIDE entry additionally contains analyses that are not a part of this study. 


\section{References}

8941 Goedert, M., Eisenberg, D. S. \& Crowther, R. A. Propagation of Tau aggregates and

$895 \quad$ neurodegeneration. Annu Rev Neurosci 40, 189-210 (2017).

8962 Strang, K. H., Golde, T. E. \& Giasson, B. I. MAPT mutations, tauopathy, and mechanisms of

897 neurodegeneration. Laboratory Investigation 99, 912-928 (2019).

8983 Lewis, J. et al. Neurofibrillary tangles, amyotrophy and progressive motor disturbance in mice

899 expressing mutant (P301L) Tau protein. Nat Genet 25, 402-405 (2000).

9004 Allen, B. et al. Abundant Tau filaments and nonapoptotic neurodegeneration in transgenic mice

$901 \quad$ expressing human P301S Tau protein. J Neurosci 22, 9340-9351 (2002).

9025 Jucker, M. \& Walker, L. C. Propagation and spread of pathogenic protein assemblies in

903 neurodegenerative diseases. Nat Neurosci 21, 1341-1349 (2018).

9046 Vaquer-Alicea, J. \& Diamond, M. I. Propagation of protein aggregation in neurodegenerative 905 diseases. Annual review of biochemistry 88, 785-810 (2019).

9067 DeVos, S. L. et al. Tau reduction prevents neuronal loss and reverses pathological Tau deposition $907 \quad$ and seeding in mice with tauopathy. Sci Transl Med 9, 374 (2017).

9088 Polydoro, M. et al. Reversal of neurofibrillary tangles and Tau-associated phenotype in the 909 rTgTauEC model of early Alzheimer's disease. J Neurosci 33, 13300-13311 (2013).

9109 Doyle, S. M., Genest, O. \& Wickner, S. Protein rescue from aggregates by powerful molecular $911 \quad$ chaperone machines. Nat Rev Mol Cell Biol 14, 617-629 (2013). Mogk, A., Bukau, B. \& Kampinga, H. H. Cellular handling of protein aggregates by disaggregation machines. Mol Cell 69, 214-226 (2018).

915 Nillegoda, N. B., Wentink, A. S. \& Bukau, B. Protein disaggregation in multicellular organisms. Trends Biochem Sci 43, 285-300 (2018).

917 Faust, O. et al. HSP40 proteins use class-specific regulation to drive HSP70 functional diversity. Nature 587, 489-494 (2020). Wentink, A. S. et al. Molecular dissection of amyloid disaggregation by human HSP70. Nature 587, 483-488 (2020).

14 Shorter, J. The mammalian disaggregase machinery: Hsp110 synergizes with Hsp70 and Hsp40 to catalyze protein disaggregation and reactivation in a cell-free system. PLoS One 6, e26319, (2011). Nachman, E. et al. Disassembly of Tau fibrils by the human Hsp70 disaggregation machinery generates small seeding-competent species. J Biol Chem 295, 9676-9690 (2020).

16 Schneider, M. M. et al. The Hsc70 disaggregation machinery removes monomer units directly from $\alpha$-synuclein fibril ends. Nature Communications 12, 5999 (2021). Gao, X. et al. Human Hsp70 disaggregase reverses Parkinson's-linked alpha-Synuclein amyloid fibrils. Mol Cell 59, 781-793 (2015).

930 Bodnar, N. O. \& Rapoport, T. A. Molecular
ATPase complex. Cell 169, 722-735 (2017).

932 ATPase. Mol Cell, 82(3), 570-584 (2021).

936 Darwich, N. F. et al. Autosomal dominant VCP hypomorph mutation impairs disaggregation of PHF-tau. Science, eaay8826 (2020).

938 Kobayashi, T., Manno, A. \& Kakizuka, A. Involvement of valosin-containing protein (VCP)/p97 in the formation and clearance of abnormal protein aggregates. Genes Cells 12, 889-901 (2007). Gwon, Y. et al. Ubiquitination of G3BP1 mediates stress granule disassembly in a context-specific manner. Science 372, eabf6548 (2021). 
951

952

953

954

955

956

957

958

959

960

961

962

963

964

965

966

967

968

969

970

971

972

973

974

975

976

977

978

979

980

981

982

983

984

985

23 Buchan, J. R., Kolaitis, R.-M., Taylor, J. P. \& Parker, R. Eukaryotic stress granules are cleared by autophagy and Cdc48/VCP function. Cell 153, 1461-1474 (2013).

24 van den Boom, J. \& Meyer, H. VCP/p97-mediated unfolding as a principle in protein homeostasis and signaling. Mol Cell 69, 182-194 (2018).

25 Olszewski, M. M., Williams, C., Dong, K. C. \& Martin, A. The Cdc48 unfoldase prepares wellfolded protein substrates for degradation by the $26 \mathrm{~S}$ proteasome. Communications Biology 2, 29 (2019).

26 Meyer, H. \& Weihl, C. C. The VCP/p97 system at a glance: connecting cellular function to disease pathogenesis. Journal of Cell Science 127, 3877 (2014).

27 Johnson, J. O. et al. Exome sequencing reveals VCP mutations as a cause of familial ALS. Neuron 68, 857-864 (2010).

28 Schroder, R. et al. Mutant valosin-containing protein causes a novel type of frontotemporal dementia. Annals of neurology 57, 457-461 (2005).

29 Watts, G. D. et al. Inclusion body myopathy associated with Paget disease of bone and frontotemporal dementia is caused by mutant valosin-containing protein. Nat Genet 36, 377381 (2004).

30 Blythe, E. E., Olson, K. C., Chau, V. \& Deshaies, R. J. Ubiquitin- and ATP-dependent unfoldase activity of P97/VCP*NPLOC4*UFD1L is enhanced by a mutation that causes multisystem proteinopathy. Proc Natl Acad Sci U S A 114, E4380-E4388 (2017).

31 Manno, A., Noguchi, M., Fukushi, J., Motohashi, Y. \& Kakizuka, A. Enhanced ATPase activities as a primary defect of mutant valosin-containing proteins that cause inclusion body myopathy associated with Paget disease of bone and frontotemporal dementia. Genes Cells 15, 911-922 (2010).

32 Blythe, E. E., Gates, S. N., Deshaies, R. J. \& Martin, A. Multisystem Proteinopathy mutations in VCP/p97 increase NPLOC4.UFD1L binding and substrate processing. Structure 27, 1820-1829 (2019).

33 Ritz, D. et al. Endolysosomal sorting of ubiquitylated caveolin-1 is regulated by VCP and UBXD1 and impaired by VCP disease mutations. Nature Cell Biology 13, 1116-1123 (2011).

34 Tresse, E. et al. VCP/p97 is essential for maturation of ubiquitin-containing autophagosomes and this function is impaired by mutations that cause IBMPFD. Autophagy 6, 217-227 (2010).

35 Barghorn, S., Davies, P. \& Mandelkow, E. Tau paired helical filaments from Alzheimer's disease brain and assembled in vitro are based on beta-structure in the core domain. Biochemistry 43, 1694-1703 (2004).

36 Crowther, T., Goedert, M. \& Wischik, C. M. The repeat region of microtubule-associated protein Tau forms part of the core of the paired helical filament of Alzheimer's disease. Ann Med 21, 127-132 (1989).

37 Sanders, D. W. et al. Distinct Tau prion strains propagate in cells and mice and define different tauopathies. Neuron 82, 1271-1288 (2014).

38 Frost, B., Ollesch, J., Wille, H. \& Diamond, M. I. Conformational diversity of wild-type Tau fibrils specified by templated conformation change. J Biol Chem 284, 3546-3551 (2009).

39 Schmued, L. et al. Introducing Amylo-Glo, a novel fluorescent amyloid specific histochemical tracer especially suited for multiple labeling and large scale quantification studies. Journal of Neuroscience Methods 209, 120-126 (2012).

40 Falcon, B. et al. Structures of filaments from Pick's disease reveal a novel Tau protein fold. Nature 561, 137-140 (2018).

41 Falcon, B. et al. Novel Tau filament fold in chronic traumatic encephalopathy encloses hydrophobic molecules. Nature 568, 420-423 (2019). 


\footnotetext{
$98642 \quad$ Fitzpatrick, A. W. P. et al. Cryo-EM structures of Tau filaments from Alzheimer's disease. Nature 
103364 Sontag, E. M., Samant, R. S. \& Frydman, J. Mechanisms and functions of spatial protein quality

1034

1035

1036

1037

1038

1039

1040

1041

1042

1043

1044

1045

1046

1047

1048

1049

1050

1051

1052

1053

1054

1055

1056

1057

1058

1059

1060

1061

1062

1063

1064

1065

1066

1067

1068

1069

1070

1071

1072

1073

1074

1075

1076

1077 control. Annual review of biochemistry 86, 97-122 (2017).

65 Cliffe, R. et al. Filamentous aggregates are fragmented by the proteasome holoenzyme. Cell Rep 26, 2140-2149 (2019).

66 Higashiyama, H. et al. Identification of ter94, Drosophila VCP, as a modulator of polyglutamineinduced neurodegeneration. Cell Death Differ 9, 264-273 (2002).

67 Khosravi, B. et al. Cell-to-cell transmission of C9orf72 poly-(Gly-Ala) triggers key features of ALS/FTD. EMBO J 39, e102811 (2020).

68 Jackrel, M. E. et al. Potentiated Hsp104 variants antagonize diverse proteotoxic misfolding events. Cell 156, 170-182 (2014).

69 Shorter, J. Engineering therapeutic protein disaggregases. Mol Biol Cell 27, 1556-1560 (2016).

70 VerPlank, J. J. S., Tyrkalska, S. D., Fleming, A., Rubinsztein, D. C. \& Goldberg, A. L. cGMP via PKG activates $26 \mathrm{~S}$ proteasomes and enhances degradation of proteins, including ones that cause neurodegenerative diseases. Proc Natl Acad Sci U S A 117, 14220-14230 (2020).

$71 \mathrm{Gu}, \mathrm{X}$. et al. SAMTOR is an S-adenosylmethionine sensor for the mTORC1 pathway. Science 358, 813-818 (2017).

72 Schmittgen, T. D. \& Livak, K. J. Analyzing real-time PCR data by the comparative C(T) method. Nat Protoc 3, 1101-1108 (2008).

73 Guo, Q. et al. In situ structure of neuronal C9orf72 poly-GA aggregates reveals proteasome recruitment. Cell 172, 696-705 (2018).

74 Rigort, A. et al. Focused ion beam micromachining of eukaryotic cells for cryoelectron tomography. Proc Natl Acad Sci U S A 109, 4449-4454 (2012).

75 Mastronarde, D. N. Automated electron microscope tomography using robust prediction of specimen movements. J Struct Biol 152, 36-51 (2005).

76 Fukuda, Y., Laugks, U., Lučić, V., Baumeister, W. \& Danev, R. Electron cryotomography of vitrified cells with a Volta phase plate. J Struct Biol 190, 143-154 (2015).

$77 \mathrm{Li}, \mathrm{X}$. et al. Electron counting and beam-induced motion correction enable near-atomicresolution single-particle cryo-EM. Nat Methods 10, 584-590 (2013).

78 Kremer, J. R., Mastronarde, D. N. \& McIntosh, J. R. Computer visualization of three-dimensional image data using IMOD. J Struct Biol 116, 71-76 (1996).

79 Rigort, A. et al. Automated segmentation of electron tomograms for a quantitative description of actin filament networks. J Struct Biol 177, 135-144 (2012).

80 Martinez-Sanchez, A., Garcia, I., Asano, S., Lucic, V. \& Fernandez, J. J. Robust membrane detection based on tensor voting for electron tomography. J Struct Biol 186, 49-61 (2014).

81 Ong, S. E. et al. Stable isotope labeling by amino acids in cell culture, SILAC, as a simple and accurate approach to expression proteomics. Mol Cell Proteomics 1, 376-386 (2002).

82 Wiśniewski, J. R., Zielinska, D. F. \& Mann, M. Comparison of ultrafiltration units for proteomic and $\mathrm{N}$-glycoproteomic analysis by the filter-aided sample preparation method. Anal Biochem 410, 307-309 (2011).

83 Banning, C. et al. A flow cytometry-based FRET assay to identify and analyse protein-protein interactions in living cells. PLoS One 5, e9344 (2010).

84 Perez-Riverol, Y. et al. The PRIDE database and related tools and resources in 2019: improving support for quantification data. Nucleic Acids Res 47, D442-d450 (2019). 
1078

1079

1080

1081

1082

1083

1084

1085

1086

1087

1088

1089

1090

1091

1092

1093

1094

1095

1096

1097

1098

1099

\section{Acknowledgements}

We thank Ralf Zenke, Markus Oster, Giovanni Cardone and Martin Spitaler from the MPIB Imaging Facility for assistance with confocal microscopy, image analysis and flow cytometry, and Albert Ries for assistance with mass spectrometry. We acknowledge Gopal Jayaraj for generation of the Fluc-GFP cell line and Alonso Izzat Carvajal Alvarez for help with native PAGE analysis. The research leading to these results has received funding from the European Commission under Grant FP7 GA ERC-2012-SyG_318987-ToPAG (I.S., R.K., P.Y.-C., I.D., R.F.-B., F.U.H. and M.S.H.), Marie Skłodowska-Curie grant (agreement no. 749370) (S.G.), the Deutsche Forschungsgemeinschaft (DFG, German Research Foundation) under Germany’s Excellence Strategy within the framework of the Munich Cluster for Systems Neurology (EXC 2145 SyNergy - ID 390857198) (V.A.T., F.U.H. and M.S.H.) and MBExC (EXC 2067/1 — ID 390729940) (R.F.-B.), as well as by the joint efforts of The Michael J. Fox Foundation for Parkinson's Research (MJFF) and the Aligning Science Across Parkinson's (ASAP) initiative. MJFF administers the grant ASAP-000282 on behalf of ASAP and itself. M.S.H. acknowledges the funding from Alzheimer Nederland (Grant Number WE.03-2020-12).

\section{Author contributions}

I.S. designed and performed most experiments. P.Y. performed seeding experiments. M.D.P. performed neuronal cultures. Q.G. and V.A.T. carried out cryo-electron tomography of Tau aggregates in TauRD-Y* cells and primary neurons, respectively. R.K. performed mass spectrometry analysis. S.G. helped with initial experiments and quantified inclusion size. H.H. performed mRNA analysis. I.D. supervised experiments with neuronal cultures. R.F.B. and W.B. supervised cryo-electron tomography experiments. D.W.S. and M.I.D. provided cell lines, 
1100 protocols and contributed to the interactome analysis. F.U.H. and M.S.H. initiated and 1101 supervised the project and wrote the manuscript with input from I.S. and the other authors.

1102

1103 Competing interests

1104 Authors declare no competing interests.

1105

1106 